\title{
Nonexpansive Mappings on New Premodular Special Space of Sequences
}

\author{
Awad A. Bakery $\mathbb{D}^{1,2}$ and OM Kalthum S. K. Mohamed $\mathbb{D}^{1,3}$ \\ ${ }^{1}$ University of Jeddah, College of Science and Arts at Khulis, Department of Mathematics, Jeddah, Saudi Arabia \\ ${ }^{2}$ Department of Mathematics, Faculty of Science, Ain Shams University, Cairo, Abbassia, Egypt \\ ${ }^{3}$ Academy of Engineering and Medical Sciences, Department of Mathematics, Khartoum, Sudan
}

Correspondence should be addressed to OM Kalthum S. K. Mohamed; om_kalsoom2020@yahoo.com

Received 13 November 2021; Accepted 15 December 2021; Published 4 January 2022

Academic Editor: Sun Young Cho

Copyright (c) 2022 Awad A. Bakery and OM Kalthum S. K. Mohamed. This is an open access article distributed under the Creative Commons Attribution License, which permits unrestricted use, distribution, and reproduction in any medium, provided the original work is properly cited.

\begin{abstract}
For different premodular, which is a generalization of modular, defined by weighted Orlicz sequence space and its prequasi operator ideal, we have examined the existence of a fixed point for both Kannan contraction and nonexpansive mappings acting on these spaces. Some numerous numerical experiments and practical applications are presented to support our results.
\end{abstract}

\section{Introduction}

The spaces of all, bounded, $\mathrm{r}$-absolutely summable, and null sequences of real numbers will be denoted throughout the article by $\mathbf{R}^{\mathscr{E}^{+}}, \ell_{\infty}, \ell_{r}$, and $c_{0}$, respectively, where $\mathscr{Z}^{+}$is the set of nonnegative integers.

Definition 1. [1, 2] An Orlicz function is a function $M:[0, \infty) \longrightarrow[0, \infty)$, which is continuous and strictly increasing with $M(0)=0, \quad M(v)>0$ for $v>0$, and $M(v) \longrightarrow \infty$, as $v \longrightarrow \infty$.

Definition 2. An Orlicz function $M$ is said to satisfy $\Delta_{2}$-condition for every values of $v \geq 0$, if there is $k>0$, such that $M(2 v) \leq k M(v)$. The $\Delta_{2}$-condition is equivalent to $M(l v) \leq k l M(v)$ for every values of $l>1$ and $v$.

Lindentrauss and Tzafriri [3] utilized the idea of a convex Orlicz function to define Orlicz sequence space:

$$
\begin{aligned}
\ell_{M} & =\left\{v \in \mathbf{R}^{\mathscr{X}^{+}}: \rho(\omega v)<\text { of for some } \omega>0\right\}, \text { where } \rho(v) \\
& =\sum_{y=0}^{\infty} M\left(\left|v_{y}\right|\right) .
\end{aligned}
$$

$\left(\ell_{M},\|\|.\right)$ is a Banach space with the Luxemburg norm:

$$
\|v\|=\inf \left\{\omega>0: \rho\left(\frac{v}{\omega}\right) \leq 1\right\} .
$$

Every Orlicz sequence space contains a subspace that is isomorphic to $c_{0}$ or $\ell_{r}$, for some $1 \leq r<\infty$ ([4], Theorem 4.a.9). The space of all bounded linear operators from a Banach space $\mathfrak{X}$ into a Banach space $\mathfrak{Y}$ will be denoted by $\mathscr{B}(\mathfrak{X}, \mathfrak{Y})$ and if $\mathfrak{X}=\mathfrak{Y}$, we write $\mathscr{B}(\mathfrak{X})$. $e_{x}=\{0,0, \ldots, 1,0,0, \ldots\}$, while 1 lies in the $x^{\text {th }}$ place, with $x \in \mathscr{Z}^{+}$.

Definition 3. [5] An s-number function is a mapping from $\mathscr{B}(\mathfrak{X}, \mathfrak{Y})$ into $[0, \infty)^{\mathscr{E}^{+}}$which transforms every map $H \in \mathscr{B}(\mathfrak{X}, \mathfrak{Y})$ to $\left(s_{x}(H)\right)_{x=0}^{\infty}$ satisfying the next conditions:

(i) $\|H\|=s_{0}(H) \geq s_{1}(H) \geq s_{2}(H) \geq \cdots \geq 0$, for every $H \in \mathscr{B}(\mathfrak{X}, \mathfrak{Y})$,

(ii) $s_{y+x-1}\left(H_{1}+H_{2}\right) \leq s_{y}\left(H_{1}\right)+s_{x}\left(H_{2}\right)$, for every $H_{1}, H_{2} \in \mathscr{B}(\mathfrak{X}, \mathfrak{Y})$, and $y, x \in \mathscr{Z}^{+}$,

(iii) ideal property: $s_{x}(U T H) \leq\|U\| s_{x}(T)\|H\|$, for every $H \in \mathscr{B}\left(\mathfrak{X}_{0}, \mathfrak{X}\right), T \in \mathscr{B}(\mathfrak{X}, \mathfrak{Y})$ and $U \in \mathscr{B}\left(\mathfrak{Y}, \mathfrak{Y}_{0}\right)$, where $\mathfrak{X}_{0}$ and $\mathfrak{Y}_{0}$ are any two Banach spaces, 
(iv) for $H \in \mathscr{B}(\mathfrak{X}, \mathfrak{Y})$ and $\omega \in \mathbf{R}$, we have $s_{x}(\omega H)=|\omega| s_{x}(H)$,

(v) rank property: If $\operatorname{rank}(H) \leq x$, then $s_{x}(H)=0$, for all $H \in \mathscr{B}(\mathfrak{X}, \mathfrak{Y})$,

(vi) norming property: $s_{l \geq x}\left(I_{x}\right)=0$ or $s_{l<x}\left(I_{x}\right)=1$, where $I_{x}$ explains the unit map on the $x$-dimensional Hilbert space $\ell_{2}^{x}$.

The $x$ th approximation number, $\alpha_{x}(W)$, is defined as $\alpha_{x}(H)=\inf \{\|H-Y\|: Y \in \mathscr{B}(\mathfrak{X}, \mathfrak{Y})$ and $\operatorname{rank}(Y) \leq x\}$.

Notations 1 . The sets $S_{W}, S_{W}(\mathfrak{X}, \mathfrak{Y}), S_{W}^{\text {app }}$, and $S_{W}^{\text {app }}(\mathfrak{X}, \mathfrak{Y})$ (cf. [6]) are defined as follows:

$$
\begin{aligned}
S_{W} & :=\left\{S_{W}(\mathfrak{X}, \mathfrak{Y})\right\}, \text { where } S_{W}(\mathfrak{X}, \mathfrak{Y}) \\
& :=\left\{H \in \mathscr{B}(\mathfrak{X}, \mathfrak{Y}):\left(\left(s_{x}(H)\right)_{x=0}^{\infty} \in W\right\} .\right. \text { Also } \\
S_{W}^{\text {app }} & :=\left\{S_{W}^{\text {app }}(\mathfrak{X}, \mathfrak{Y})\right\}, \text { where } S_{W}^{\text {app }}(\mathfrak{X}, \mathfrak{Y}) \\
& :=\left\{H \in \mathscr{B}(\mathfrak{X}, \mathfrak{Y}):\left(\left(\alpha_{x}(H)\right)_{x=0}^{\infty} \in W\right\} .\right.
\end{aligned}
$$

Fixed point theory, Banach space geometry, normal series theory, ideal transformations, and approximation theory are all examples of ideal operator theorems and summability. Faried and Bakery [6] established the concept of a prequasi operator ideal that encapsulates the quasi operator ideal. Bakery and Abou Elmatty investigated the sufficient (but not necessary) conditions on $\ell(\gamma, r)$ that allowed $S_{\ell(\gamma, r)}$ to build a simple Banach prequasi operator ideal in [7]. For varied weights and powers, the prequasi operator ideal $S_{\ell(\gamma, r)}^{\mathrm{app}}$ was once rigorously contained and small prequasi operator ideal. Several mathematicians were able to investigate many extensions for contraction maps defined on the space or on the space itself thanks to the Banach fixed point theorem [8]. Kannan [9] investigated an example of a class of operators that perform the same fixed point actions as contractions but are not continuous. Kannan operators in modular vector spaces have only been described by Ghoncheh [10]. He demonstrated the existence of a Kannan mapping fixed point in complete modular spaces with Fatou property. For more details on Kannan's fixed point theorems and modular vector spaces (see [11-14]). Bakery and Mohamed [15] introduced the concept of the prequasi norm on $\ell^{\left(\left(r_{a}\right)\right)}$ with variable exponent in $(0,1]$. They looked at the Fatou property of different prequasi norms on $\ell^{\left(\left(r_{a}\right)\right)}$, as well as the sufficient requirements on $\ell^{\left(\left(r_{a}\right)\right)}$ with the definite prequasi norm to construct prequasi Banach and closed space. They also demonstrated the existence of a fixed point of Kannan prequasi norm contraction maps on $\ell^{\left(\left(r_{a}\right)\right)}$ and the prequasi Banach operator ideal constructed by $\ell^{\left(\left(r_{a}\right)\right)}$ and $s$-numbers. Recently, Reich and Zaslavski [16] showed the existence of a unique fixed point for nonlinear contractive self-mappings of a nonbounded closed subset of a Banach space. They extended this conclusion to contractive mappings, which map into a Banach space a closed subset of the space. For nonexpansive mappings defined by an intersection of a finite number of closed bounded and convex nonempty subsets in Banach spaces, Dehici and Redjel [17] obtained certain fixed point results. According to Bendahmane and Bendoukha [18], a $(p, q)$-metric space is a generalization of the metric and $S$-metric spaces. They equipped them a Hausdorff topology and specified several fundamental features. Several wellknown findings from fixed point theory are generalized to these new spaces. The paper is structured as follows: we present conditions on the weighted Orlicz sequence space $\left(\ell_{M}(\lambda)\right)_{\mu}$, under definite prequasi norm of $\mu$ to construct prequasi Banach and closed sequence space in Section 3. The Fatou property of $\ell_{M}(\lambda)$ has been investigated for various prequasi norms. In Section 4, the existence of fixed point for Kannan $\mu$-contraction mapping acting on $\left(\ell_{M}(\lambda)\right)_{\mu}$ equipped with different prequasi norms are presented. Several numerical experiments are shown to demonstrate our results. In Section 5, the conditions for which the space $\left(\ell_{M}(\lambda)\right)_{\mu}$ satisfies the property $(R)$ and has the $\mu$-normal structure property are presented. The existence of a fixed point of Kannan prequasi norm nonexpansive mapping on $\left(\ell_{M}(\lambda)\right)_{\mu}$ has been given. In Section 6 , we explain the existence of a fixed point of Kannan prequasi norm contraction

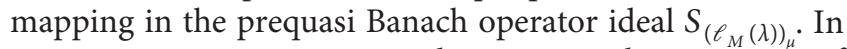
Section 7 , we give some applications to the existence of solutions of summable equations.

\section{Definitions and Preliminaries}

Here and after, the space of all functions $\mu: Y \longrightarrow[0, \infty)$ is $[0, \infty)^{Y}, \theta$ is the zero vector of $Y,[x / 2]$ is the integral part of $x / 2, F$ is the space of finite sequences, and $\mathscr{B}$ is the class of each bounded linear mapping between any two Banach spaces. Nakano [19] introduced the concept of modular vector spaces.

Definition 4. Let $Y$ be a vector space. A function $\mu \in[0, \infty]^{Y}$ is called modular if the following conditions hold:

(i) If $\beta \in Y, \beta=\theta \Leftrightarrow \mu(\beta)=0$ and $\mu(\beta) \geq 0$,

(ii) if $\beta \in Y$ and $|\omega|=1$, then $\mu(\omega \beta)=\mu(\beta)$,

(iii) assume $\quad \beta, \eta \in Y \quad$ and $\omega \in[0,1]$, then $\mu(\omega \beta+(1-\omega) \eta) \leq \mu(\beta)+\mu(\eta)$.

The concept of premodular vector spaces, which is more general than modular vector spaces.

Definition 5. [6] The linear space of sequences $Y$ is said to be a special space of sequences (sss), if:

(1) $\left\{e_{x}\right\}_{x \in \mathscr{L}^{+}} \subseteq Y$,

(2) $Y$ is solid, i.e., for $\beta=\left(\beta_{x}\right) \in \mathbf{R}^{\mathscr{E}^{+}}, \eta=\left(\eta_{x}\right) \in Y$ and $\left|\beta_{x}\right| \leq\left|\eta_{x}\right|$, for all $x \in \mathscr{Z}^{+}$, then $\beta \in Y$,

(3) If $\left(\beta_{x}\right)_{x=0}^{\infty} \in Y$, then $\left(\beta_{[x / 2]}\right)_{x=0}^{\infty} \in Y$.

Definition 6. [6] A subclass $Y_{\mu}$ of $Y$ is called a premodular (sss), if we have $\mu \in[0, \infty)^{Y^{\mu}}$ that satisfies the following conditions: 
(i) When $\beta \in Y, \beta=\theta \Leftrightarrow \mu(\beta)=0$,

(ii) For every $\beta \in Y$ and $\omega \in \mathbf{R}$, then there is $B \geq 1$ with $\mu(\omega \beta) \leq B|\omega| \mu(\beta)$

(iii) $\mu(\beta+\eta) \leq J(\mu(\beta)+\mu(\eta))$, for all $\beta, \eta \in Y$, holds for some $J \geq 1$,

(iv) If $x \in \mathscr{Z}^{+}$and $\left|\beta_{x}\right| \leq\left|\eta_{x}\right|$, then $\mu\left(\left(\beta_{x}\right)\right) \leq \mu\left(\left(\eta_{x}\right)\right)$,

(v) For some $J_{0} \geq 1$, we have $\mu\left(\left(\beta_{x}\right)\right) \leq \mu\left(\left(\beta_{[x / 2]}\right)\right) \leq J_{0} \mu\left(\left(\beta_{x}\right)\right)$,

(vi) $\bar{F}=Y_{\mu}$,

(vii) There exists $\quad \varsigma>0$ such that $\mu(\omega, 0,0,0, \ldots) \geq \varsigma|\omega| \mu(1,0,0,0, \ldots)$, for all $\omega \in \mathbf{R}$.

Example 1. The function $\mu(\beta)=\left(\sum_{x \in \mathscr{Z}^{+}} \sqrt[5]{\left|\beta_{x}\right|}\right)^{5}$ is a premodular (not a modular) on the vector space $\ell_{1 / 5}$. As for every $\beta, \eta \in \ell_{1 / 5}$, one has

$$
\mu\left(\frac{\beta+\eta}{2}\right)=\left(\sum_{x \in \mathscr{Z}^{+}} \sqrt[5]{\left|\frac{\beta_{x}+\eta_{x}}{2}\right|}\right)^{5} \leq 8(\mu(\beta)+\mu(\eta)) .
$$

Definition 7. [15] Suppose $Y$ is a (sss). The function $\mu \in[0, \infty)^{Y}$ is said to be prequasi norm on $Y$, if it holds the settings (i), (ii), and (iii) of Definition 6.

Theorem 1. [15] Let $Y$ be a premodular (sss), then it is prequasi normed (sss).

Theorem 2. [15] $Y$ is a prequasi normed (sss), when it is quasi-normed (sss).

Definition 8. [20]

(i) The prequasi norm $\mu$ on $X_{\mu}$ is said to be $\mu$-convex, when $\mu(\omega \beta+(1-\omega) \eta) \leq \omega \mu(\beta)+(1-\omega) \mu(\eta)$, for all $\omega \in[0,1]$ and $\beta, \eta \in X_{\mu}$.

(ii) $\left\{\beta_{x}\right\}_{x \in \mathscr{Z}^{+}} \subseteq(X)_{\mu}$ is $\mu$-convergent to $\beta \in(X)_{\mu}$, if and only if, $\lim _{x \rightarrow \infty} \mu\left(\beta_{x}-\beta\right)=0$. If the $\mu$-limit exists, hence it is unique.

(iii) $\left\{\beta_{x}\right\}_{x \in \mathscr{Z}^{+}} \subseteq(X)_{\mu}$ is $\mu$-Cauchy, if $\lim _{x, h \rightarrow \infty}$ $\mu\left(\beta_{x}-\beta_{h}\right)=0$.

(iv) $\Phi \subset(X)_{\mu}$ is $\mu$-closed, if for every $\mu$-converging $\left\{\beta_{x}\right\}_{x \in \mathscr{Z}^{+}} \subset \Phi$ to $\beta$, then $\beta \in \Phi$.

(v) $\Phi \subset(X)_{\mu} \quad$ is $\quad \mu$-bounded, $v_{\mu}(\Phi)=\sup \{\mu(\beta-\eta): \beta, \eta \in \Phi\}<\infty$.

(vi) The $\mu$-ball of radius $r \geq 0$ and center $\beta$, for every $\beta \in(X)_{\mu}$, is defined as

$$
\mathscr{B}_{\mu}(\beta, r)=\left\{\eta \in(X)_{\mu}: \mu(\beta-\eta) \leq r\right\} .
$$

(vii) A prequasi norm $\mu$ on $X$ satisfies the Fatou property, if for every sequence $\left\{\eta^{x}\right\} \subseteq(X)_{\mu}$ with $\lim _{x \rightarrow \infty} \mu\left(\eta^{x}-\eta\right)=0$ and any $\beta \in(X)_{\mu}$, we have $\mu(\beta-\eta) \leq \sup _{m} \inf _{x \geq m} \mu\left(\beta-\eta^{x}\right)$.

Recall that the $\mu$-balls are $\mu$-closed under the Fatou property.
Definition 9. [21] A subclass $\mathscr{G}$ of $\mathscr{B}$ is called an operator ideal, if every vector $\mathscr{G}(\mathfrak{X}, \mathfrak{Y})=\mathscr{G} \cap \mathscr{B}(\mathfrak{X}, \mathfrak{Y})$ holds the following conditions:

(i) $I_{\gamma} \in \mathscr{G}$, where $\gamma$ indicates Banach space of one dimension.

(ii) The space $\mathscr{G}(\mathfrak{X}, \mathfrak{Y})$ is linear over $\mathbf{R}$.

(iii) If $\quad H \in \mathscr{B}\left(\mathfrak{X}_{0}, \mathfrak{X}\right), \quad T \in \mathscr{G}(\mathfrak{X}, \mathfrak{Y})$, $\quad$ and $V \in \mathscr{B}\left(\mathfrak{Y}, \mathfrak{Y}_{0}\right)$, then $V T H \in \mathscr{G}\left(\mathfrak{X}_{0}, \mathfrak{Y}_{0}\right)$, where $\mathfrak{X}_{0}$ and $\mathfrak{V}_{0}$ are normed spaces.

Recall that the quasi operator ideals are a special case of the prequasi operator ideals.

Definition 10. [6] A function $\Upsilon \in[0, \infty)^{\mathscr{G}}$ is said to be a prequasi norm on the ideal $\mathscr{G}$ if the following conditions verify:

(1) Suppose $H \in \mathscr{G}(\mathfrak{X}, \mathfrak{Y}), \Upsilon(H) \geq 0$ and $\Upsilon(H)=0$, if and only if, $H=0$,

(2) there exists $D \geq 1$ such that $\Upsilon(\omega H) \leq D|\omega| \Upsilon(H)$, for every $H \in \mathscr{G}(\mathfrak{X}, \mathfrak{Y})$ and $\omega \in \mathbf{R}$,

(3) we have $J \geq 1$ so that $\Upsilon\left(H_{1}+H_{2}\right) \leq J\left[\Upsilon\left(H_{1}\right)+\Upsilon\left(H_{2}\right)\right]$, for all $H_{1}, H_{2} \in \mathscr{G}(\mathfrak{X}, \mathfrak{Y})$,

(4) we get $\omega \geq 1$ so that if $H \in \mathscr{B}\left(\mathfrak{X}_{0}, \mathfrak{X}\right), T \in \mathscr{G}(\mathfrak{X}, \mathfrak{Y})$, and $V \in \mathscr{B}\left(\mathfrak{Y}, \mathfrak{Y}_{0}\right)$, then $\Upsilon(V T H) \leq \omega\|V\| \Upsilon(T)\|H\|$.

Theorem 3. [15] The function $\Upsilon(H)=\mu\left(s_{x}(H)\right)_{x=0}^{\infty}$ is a prequasi norm on $S_{Y_{\mu}}$, when $Y_{\mu}$ is a premodular (sss).

Theorem 4. [6] If $\Upsilon$ is a quasi norm on the ideal $\mathscr{G}$, then $\Upsilon$ is a prequasi norm on the ideal $\mathscr{G}$.

Lemma 1. $[22,23]$ Assume $M:(0, \infty) \longrightarrow[0, \infty)$ is a continuous function and strictly increasing with $\lim _{x \rightarrow 0} M(x)=0$, and if the functions $M(x)$ and $\ln \left(M\left(e^{x}\right)\right)$ are convex on $[0, \infty)$, then

$$
\begin{aligned}
M^{-1}\left(\sum_{x=0}^{\infty} \lambda_{x} M\left(\beta_{x}+\eta_{x}\right)\right) \leq & M^{-1}\left(\sum_{x=0}^{\infty} \lambda_{x} M\left(\beta_{x}\right)\right) \\
& +M^{-1}\left(\sum_{x=0}^{\infty} \lambda_{x} M\left(\eta_{x}\right)\right) .
\end{aligned}
$$

$$
\lambda_{x}, \beta_{x}, \eta_{x} \in[0, \infty) \text {, for all } x \in \mathscr{Z}^{+} \text {and } \sum_{x=0}^{\infty} \lambda_{x}=1 \text {. }
$$

\section{Main Results}

3.1. Properties of Different Prequasi Norms. In this section, we have studied some topological structures and the Fatou property of the weighted Orlicz sequence space, $\ell_{M}(\lambda)$, for various prequasi norms.

Lemma 2. If $M$ is a concave Orlicz function, then $M(x+y) \leq M(x)+M(y)$, for all $x, y \in[0, \infty)$.

Proof. It is easy so omitted. 
Theorem 5. $\left(\ell_{M}(\lambda)\right)_{\mu}$, where $\mu(\beta)=\sum_{y=0}^{\infty} \lambda_{y} M\left(\left|\beta_{y}\right|\right)$, for each $\beta \in \ell_{M}(\lambda)$, is a premodular (sss), if $M$ is a concave Orlicz function or convex Orlicz function satisfying $\Delta_{2}$-condition.

Proof. Suppose $M$ is a convex Orlicz function satisfying $\Delta_{2}$-condition. First, we must demonstrate that $\ell_{M}(\lambda)$ is a (sss):

(1)

(i) Let $\beta, t \in \ell_{M}(\lambda)$. As $M$ is a strictly increasing and convex function satisfying $\Delta_{2}$-condition, we get

$$
\begin{aligned}
\mu(\beta+\eta) & =\sum_{y=0}^{\infty} \lambda_{y} M\left(\left|\beta_{y}+\eta_{y}\right|\right) \\
& \leq \frac{k}{2}\left[\sum_{y=0}^{\infty} \lambda_{y} M\left(\left|\beta_{y}\right|\right)+\sum_{y=0}^{\infty} \lambda_{y} M\left(\left|\eta_{y}\right|\right)\right] \\
& =\frac{k}{2}(\mu(\beta)+\mu(\eta))<\infty,
\end{aligned}
$$

this implies $\beta+\eta \in \ell_{M}(\lambda)$.

(ii) Suppose $\omega \in \mathbf{R}$ and $\beta \in \ell_{M}(\lambda)$. Since $M$ satisfies $\Delta_{2}$-condition, we have

$$
\begin{aligned}
\mu(\omega \beta) & =\sum_{y=0}^{\infty} \lambda_{y} M\left(\left|\omega \beta_{y}\right|\right) \\
& \leq k|\omega| \sum_{y=0}^{\infty} \lambda_{y} M\left(\left|\beta_{y}\right|\right) \leq D|\omega| \mu(\beta)<\infty .
\end{aligned}
$$

So $\omega \beta \in \ell_{M}(\lambda)$. Therefore, from conditions 1 (i) and (ii), one has $\ell_{M}(\lambda)$ is linear. We have $e_{y} \in \ell_{M}(\lambda)$, for every $y \in \mathscr{Z}^{+}$, as

(2) Let $\left|\beta_{y}\right| \leq\left|\eta_{y}\right|$, for every $y \in \mathscr{Z}^{+}$and $\eta \in \ell_{M}(\lambda)$. Since $M$ is a nondecreasing function, then

$$
\begin{aligned}
\mu(\beta) & =\sum_{y=0}^{\infty} \lambda_{y} M\left(\left|\beta_{y}\right|\right) \\
& \leq \sum_{y=0}^{\infty} \lambda_{y} M\left(\left|\eta_{y}\right|\right)=\mu(\eta)<\infty,
\end{aligned}
$$

one has $\beta \in \ell_{M}(\lambda)$.

(3) Assume $\left(\beta_{y}\right) \in \ell_{M}(\lambda)$, we get

$$
\begin{aligned}
\mu\left(\left(\beta_{[y / 2]}\right)\right) & =\sum_{y=0}^{\infty} \lambda_{y} M\left(\left|\beta_{[y / 2]}\right|\right) \\
& \leq 2 \sum_{y=0}^{\infty} \lambda_{y} M\left(\left|\beta_{y}\right|\right)=2 \mu\left(\left(\beta_{y}\right)\right)<\infty,
\end{aligned}
$$

then $\left(\beta_{[y / 2]}\right) \in \ell_{M}(\lambda)$. Second, to prove that the functional $\mu$ on $\ell_{M}(\lambda)$ is a premodular:

(i) Obviously, $\mu(\beta) \geq 0$ and $\mu(\beta)=0 \Leftrightarrow \beta=\theta$.

(ii) There are $D=\max \{1, k\} \geq 1 \quad$ with $\mu(\omega \beta) \leq D|\omega| \mu(\beta)$, for every $\beta \in \ell_{M}(\lambda)$ and $\omega \in \mathbf{R}$. (iii) There exists $J=\max \{1, k / 2\} \geq 1 \quad$ with $\mu(\beta+\eta) \leq J(\mu(\beta)+\mu(\eta)), \quad$ for $\quad$ every $\beta, \eta \in \ell_{M}(\lambda)$.

(iv) Follows the proof part (2).

(v) Follows from the proof part (3) that $J_{0}=2 \geq 1$.

(vi) Obviously, $\bar{F}=\ell_{M}(\lambda)$.

(vii) There exists $0<\varsigma \leq M_{0}(|\omega|) /|\omega| M_{0}(1)$, for $\omega \neq 0$ or $\varsigma>0$, for $\omega=0$ so that $\mu(\omega, 0,0,0, \ldots)$ $\geq \varsigma|\omega| \mu(1,0,0,0, \ldots)$.

If $M$ is a concave Orlicz function. By applying Lemma 2 and the parallel proof follows.

Theorem 6. If $M$ is a concave Orlicz function or convex Orlicz function satisfying $\Delta_{2}$-condition, then $\left(\ell_{M}(\lambda)\right)_{\mu}$ is a prequasi Banach (sss), where $\mu(\beta)=\sum_{y=0}^{\infty} \lambda_{y} M\left(\left|\beta_{y}\right|\right)$, for each $\beta \in \ell_{M}(\lambda)$.

Proof. Suppose $M$ is a convex Orlicz function satisfying $\Delta_{2}$-condition. By using Theorem 5 , the space $\left(\ell_{M}(\lambda)\right)_{\mu}$ is a premodular (sss). From Theorem 1 , the space $\left(\ell_{M}(\lambda)\right)_{\mu}$ is a prequasi normed (sss). To prove that $\left(\ell_{M}(\lambda)\right)_{\mu}$ is a prequasi Banach (sss), let $\beta^{r}=\left(\beta_{y}^{r}\right)_{y=0}^{\infty}$ be a Cauchy sequence in $\left(\ell_{M}(\lambda)\right)_{\mu}$. Therefore, for all $\epsilon \in(0,1)$, we have that for every $r, t \geq r_{0}$, we get

$$
\mu\left(\beta^{r}-\beta^{t}\right)=\sum_{y=0}^{\infty} \lambda_{y} M\left(\left|\beta_{y}^{r}-\beta_{y}^{t}\right|\right)<\epsilon .
$$

Hence, for $r, t \geq r_{0}$ and $y \in \mathscr{Z}^{+}$, one has $\left|\beta_{y}^{r}-\beta_{y}^{t}\right|<\epsilon$ Then $\left(\beta_{y}^{t}\right)$ is a Cauchy sequence in $\mathbf{R}$, for fixed $y \in \mathscr{Z}^{+}$. This gives $\lim _{t \rightarrow \infty} \beta_{y}^{t}=\beta_{y}^{0}$, for constant $y \in \mathscr{Z}^{+}$. Therefore, $\mu\left(\beta^{r}-\beta^{0}\right)<\epsilon$, for all $r \geq r_{0}$. To investigate that $\beta^{0} \in \ell_{M}(\lambda)$, one has $\mu\left(\beta^{0}\right)=\mu\left(\beta^{0}-\beta^{r}+\beta^{r}\right) \leq J\left(\mu\left(\beta^{r}-\beta^{0}\right)\right.$ $\left.+\mu\left(\beta^{r}\right)\right)<\infty$, so $\beta^{0} \in \ell_{M}(\lambda)$. This implies that $\left(\ell_{M}(\lambda)\right)_{\mu}$ is a prequasi Banach (sss). If $M$ is a concave Orlicz function. By applying Lemma 2 and the parallel proof follows.

Theorem 7. If $M$ is a concave Orlicz function or convex Orlicz function satisfying $\Delta_{2}$-condition, then $\left(\ell_{M}(\lambda)\right)_{\mu}$ is a prequasi closed (sss), where $\mu(\beta)=\sum_{y=0}^{\infty} \lambda_{y} M\left(\left|\beta_{y}\right|\right)$, for every $\beta \in \ell_{M}(\lambda)$.

Proof. Let $M$ be a convex Orlicz function satisfying $\Delta_{2}$-condition. According to Theorem 5 , the space $\left(\ell_{M}(\lambda)\right)_{\mu}$ is a premodular (sss). From Theorem 1 , the space $\left(\ell_{M}(\lambda)\right)_{\mu}$ is a prequasi normed (sss). To prove that $\left(\ell_{M}(\lambda)\right)_{\mu}$ is a prequasi closed (sss), suppose $\beta^{r}=\left(\beta_{y}^{r}\right)_{y=0}^{\infty} \in\left(\ell_{M}(\lambda)\right)_{\mu}$ and $\lim _{r \rightarrow \infty} \mu\left(\beta^{r}-\beta^{0}\right)=0$, hence for all $\epsilon \in(0,1)$, one has $r_{0} \in \mathscr{Z}^{+}$so that for every $r \geq r_{0}$, we have

$$
\mu\left(\beta^{r}-\beta^{0}\right)=\sum_{y=0}^{\infty} \lambda_{y} M\left(\left|\beta_{y}^{r}-\beta_{y}^{0}\right|\right)<\epsilon .
$$

Therefore, for $r \geq r_{0}$ and $y \in \mathscr{Z}^{+}$, one has $\left|\beta_{y}^{r}-\beta_{y}^{0}\right|<\epsilon$. Hence, $\left(\beta_{y}^{r}\right)$ is a convergent sequence in $\mathbf{R}$, for constant $y \in \mathscr{Z}^{+}$. So, $\lim _{r \rightarrow \infty} \beta_{y}^{r}=\beta_{y}^{0}$, for constant $y \in \mathscr{Z}^{+}$. Finally to show that $\beta^{0} \in \ell_{M}(\lambda)$, one has 


$$
\mu\left(\beta^{0}\right)=\mu\left(\beta^{0}-\beta^{r}+\beta^{r}\right) \leq J\left(\mu\left(\beta^{r}-\beta^{0}\right)+\mu\left(\beta^{r}\right)\right)<\infty .
$$

Hence, HTML translation failed. This implies that $\left(\ell_{M}(\lambda)\right)_{\mu}$ is a prequasi closed (sss). If $M$ is a concave Orlicz function, by applying Lemma 2 and the parallel proof follows.

Theorem 8. If $M$ is a convex Orlicz function satisfying $\Delta_{2}$-condition and $\ln \left(M\left(e^{x}\right)\right)$ is convex, then the function
$\mu(\beta)=M^{-1}\left(\sum_{y=0}^{\infty} \lambda_{y} M\left(\left|\beta_{y}\right|\right)\right)$ verifies the Fatou property, for all $\beta \in \ell_{M}(\lambda)$.

Proof. Assume that $\left\{\eta^{b}\right\} \subseteq\left(\ell_{M}(\lambda)\right)_{\mu}$ such that $\lim _{b \rightarrow \infty} \mu$ $\left(\eta^{b}-\eta\right)=0$. As the space $\left(\ell_{M}(\lambda)\right)_{\mu}$ is a prequasi closed space, one has $t \in\left(\ell_{M}(\lambda)\right)_{\mu}$. Hence, for every $\beta \in\left(\ell_{M}(\lambda)\right)_{\mu}$, from Lemma 1 , we have

$$
\begin{aligned}
\mu(\beta-\eta) & =M^{-1}\left(\sum_{y=0}^{\infty} \lambda_{y} M\left(\left|\beta_{y}-\eta_{y}\right|\right)\right) \leq M^{-1}\left(\sum_{y=0}^{\infty} \lambda_{y} M\left(\left|\beta_{y}-\eta_{y}^{b}\right|\right)\right)+M^{-1}\left(\sum_{y=0}^{\infty} \lambda_{y} M\left(\left|\eta_{y}^{b}-\eta_{y}\right|\right)\right) \\
& \leq \sup _{j} \inf _{b \geq j} \mu\left(\beta-\eta^{b}\right) .
\end{aligned}
$$

Hence, $\mu$ satisfies the Fatou property.

Theorem 9. If $M$ is a concave Orlicz function, then the function $\mu(\beta)=\sum_{y=0}^{\infty} \lambda_{y} M\left(\left|\beta_{y}\right|\right)$ holds the Fatou property, for all $\beta \in \ell_{M}(\lambda)$.

Proof. Suppose $\left\{\eta^{b}\right\} \subseteq\left(\ell_{M}(\lambda)\right)_{\mu} \quad$ so that $\quad \lim _{b \rightarrow \infty}$ $\mu\left(\eta^{b}-\eta\right)=0$. As the space $\left(\ell_{M}(\lambda)\right)_{\mu}$ is a prequasi closed space; hence, $\eta \in\left(\ell_{M}(\lambda)\right)_{\mu}$. As $M$ is continuous, concave and $M(0)=0$. Therefore, for every $\beta \in\left(\ell_{M}(\lambda)\right)_{\mu}$, one has

$$
\begin{aligned}
\mu(\beta-\eta)= & \sum_{y=0}^{\infty} \lambda_{y} M\left(\left|\beta_{y}-\eta_{y}\right|\right) \leq \sum_{y=0}^{\infty} \lambda_{y} M\left(\left|\beta_{y}-\eta_{y}^{b}\right|\right) \\
& +\sum_{y=0}^{\infty} \lambda_{y} M\left(\left|\eta_{y}^{b}-\eta_{y}\right|\right) \leq \sup _{j} \inf _{b \geq j} \mu\left(\beta-\eta^{b}\right) .
\end{aligned}
$$

Hence, $\mu$ satisfies the Fatou property.

Theorem 10. The function $\mu(\beta)=\sum_{y=0}^{\infty} \lambda_{y} M\left(\left|\beta_{y}\right|\right)$ does not satisfy the Fatou property, for all $\beta \in \ell_{M}(\lambda)$, if $M$ is a strictly convex Orlicz function satisfying $\Delta_{2}$-condition.

Proof. Since $M$ is a strictly convex Orlicz function satisfying $\Delta_{2}$-condition, then there exists $k>2$ such that $2 M(u)<M(2 u)<k M(u)$, for all $u \geq 0$. Let the conditions be fulfilled and $\left\{\eta^{b}\right\} \subseteq\left(\ell_{M}(\lambda)\right)_{\mu}$ with $\lim _{b \rightarrow \infty} \mu\left(\eta^{b}-\eta\right)=0$. As the space $\left(\ell_{M}(\lambda)\right)_{\mu}$ is a prequasi closed space; hence, $\eta \in\left(\ell_{M}(\lambda)\right)_{\mu}$. Since $M$ is continuous, then for any $\beta \in\left(\ell_{M}(\lambda)\right)_{\mu}$, we have

$$
\mu(\beta-\eta)=\sum_{y=0}^{\infty} \lambda_{y} M\left(\left|\beta_{y}-\eta_{y}\right|\right) \leq \frac{k}{2}\left[\sum_{y=0}^{\infty} \lambda_{y} M\left(\left|\beta_{y}-\eta_{y}^{b}\right|\right)+\sum_{y=0}^{\infty} \lambda_{y} M\left(\left|\eta_{y}^{b}-\eta_{y}\right|\right)\right] \leq \frac{k}{2} \sup _{j} \inf _{b \geq j} \mu\left(\beta-\eta^{b}\right)
$$

Therefore, $\mu$ does not hold the Fatou property.

Example 2. For every $\beta \in \ell_{M}(\lambda)$, the function $\mu(\beta)=\ln (1+$ $\left.\sum_{y=0}^{\infty} \lambda_{y}\left(e^{\left|\beta_{y}\right|}-1\right)\right)$ is a prequasi norm, not quasi, and not a norm.

Example 3. For all $\beta \in \ell_{M}(\lambda)$, the function $\mu(\beta)=\left(\sum_{y=0}^{\infty} \lambda_{y} \sqrt{\left|\beta_{y}\right|}\right)^{2}$ is a prequasi norm, quasi norm, and not a norm.

Example 4. The function $\mu(\beta)=\inf \left\{\kappa>0: \sum_{y=0}^{\infty} \lambda_{y} M\right.$ $\left.\left(\left|\beta_{y}\right| / \kappa\right) \leq 1\right\}$ is a prequasi norm, a quasi norm, and a norm on $\ell_{M}(\lambda)$.

\section{Kannan $\mu$-Contraction Operator}

We now define Kannan $\mu$-Lipschitzian mapping acting on $\left(\ell_{M}(\lambda)\right)_{\mu}$. The sufficient conditions for a fixed point of Kannan contraction mapping on $\left(\ell_{M}(\lambda)\right)_{\mu}$ under various prequasi norms are investigated.

Definition 11. An operator $H:\left(\ell_{M}(\lambda)\right)_{\mu} \longrightarrow\left(\ell_{M}(\lambda)\right)_{\mu}$ is called a Kannan $\mu$-Lipschitzian, if there exists $\nu \geq 0$, so that

$$
\mu(H \beta-H \eta) \leq \nu(\mu(H \beta-\beta)+\mu(H \eta-\eta)),
$$

for every $\beta, \eta \in\left(\ell_{M}(\lambda)\right)_{\mu}$

(1) The operator $H$ is said to be Kannan $\mu$-contraction, when $v \in[0,1 / 2)$. 
(2) The operator $H$ is said to be Kannan $\mu$-nonexpansive, whenever $v=1 / 2$.

A vector $\beta \in\left(\ell_{M}(\lambda)\right)_{\mu}$ is called a fixed point of $H$, when $H(\beta)=\beta$.

Theorem 11. If $M$ is a convex Orlicz function satisfying $\Delta_{2}$-condition and $\ln \left(M\left(e^{x}\right)\right)$ is convex, and
$H:\left(\ell_{M}(\lambda)\right)_{\mu} \longrightarrow\left(\ell_{M}(\lambda)\right)_{\mu}$ is Kannan $\mu$-contraction mapping, where $\mu(\beta)=M^{-1}\left(\sum_{y=0}^{\infty} \lambda_{y} M\left(\left|\beta_{y}\right|\right)\right)$, for all $\beta \in \ell_{M}(\lambda)$; hence, $H$ has a unique fixed point.

Proof. Assume that $\beta \in \ell_{M}(\lambda)$, one has $H^{t} \beta \in \ell_{M}(\lambda)$. Since $H$ is a Kannan $\mu$-contraction mapping, we have

$$
\begin{aligned}
& \mu\left(H^{t+1} \beta-H^{t} \beta\right) \leq \nu\left(\mu\left(H^{t+1} \beta-H^{t} \beta\right)+\mu\left(H^{t} \beta-H^{t-1} \beta\right)\right) \Rightarrow \\
& \mu\left(H^{t+1} \beta-H^{t} \beta\right) \leq \frac{\nu}{1-\nu} \mu\left(H^{t} \beta-H^{t-1} \beta\right) \leq\left(\frac{v}{1-\nu}\right)^{2} \mu\left(H^{t-1} \beta-H^{t-2} \beta\right) \leq \cdots \leq\left(\frac{v}{1-\nu}\right)^{t} \mu(H \beta-\beta) .
\end{aligned}
$$

Therefore, for every $t, v \in \mathscr{Z}^{+}$with $v>t$, then we get

$$
\begin{aligned}
\mu\left(H^{t} \beta-H^{v} \beta\right) & \leq \nu\left(\mu\left(H^{t} \beta-H^{t-1} \beta\right)+\mu\left(H^{v} \beta-H^{\nu-1} \beta\right)\right) \\
& \leq \nu\left(\left(\frac{v}{1-v}\right)^{t-1}+\left(\frac{v}{1-\nu}\right)^{\nu-1}\right) \mu(H \beta-\beta) .
\end{aligned}
$$

So, $\left\{H^{t} \beta\right\}$ is a Cauchy sequence in $\left(\ell_{M}(\lambda)\right)_{\mu}$. As the space $\left(\ell_{M}(\lambda)\right)_{\mu}$ is prequasi Banach space. Therefore, there is $\eta \in\left(\ell_{M}(\lambda)\right)_{\mu}$ such that $\lim _{t \rightarrow \infty} H^{t} \beta=\eta$. To prove that $H \eta=\eta$. As $\mu$ holds the Fatou property, we obtain

$$
\begin{aligned}
\mu(H \eta-\eta) & \leq \sup _{p} \inf _{t \geq p} \mu\left(H^{t+1} \beta-H^{t} \beta\right) \\
& \leq \sup _{p} \inf _{t \geq p}\left(\frac{v}{1-v}\right)^{t} \mu(H \beta-\beta)=0,
\end{aligned}
$$

hence $H \eta=\eta$. Hence, $\eta$ is a fixed point of $H$. To prove the uniqueness of the fixed point. For different fixed points $\zeta, \eta \in\left(\ell_{M}(\lambda)\right)_{\mu}$ of $H$. We have that

$$
\mu(\zeta-\eta) \leq \mu(H \zeta-H \eta) \leq \nu(\mu(H \zeta-\zeta)+\mu(H \eta-\eta))=0 .
$$

Therefore, $\zeta=\eta$.

Corollary 1. Let $M$ be a convex Orlicz function satisfying $\Delta_{2}$-condition and $\ln \left(M\left(e^{x}\right)\right)$ be convex, and $H:\left(\ell_{M}(\lambda)\right)_{\mu} \longrightarrow\left(\ell_{M}(\lambda)\right)_{\mu}$ be Kannan $\mu$-contraction mapping, with $\mu(\beta)=M^{-1}\left(\sum_{y=0}^{\infty} \lambda_{y} M\left(\left|\beta_{y}\right|\right)\right)$, for every $\beta \in \ell_{M}(\lambda)$, then $H$ has a unique fixed point $\zeta$ such that $\mu\left(H^{t} \beta-\zeta\right) \leq \nu(\nu / 1-\nu)^{t-1} \mu(H \beta-\beta)$.

Proof. From Theorem 11, there is a unique fixed point $\zeta$ of $H$. Hence, one has

$$
\mu\left(H^{t} \beta-\zeta\right)=\mu\left(H^{t} \beta-H \zeta\right) \leq \nu\left(\mu\left(H^{t} \beta-H^{t-1} \beta\right)+\mu(H \zeta-\zeta)\right)=\nu\left(\frac{v}{1-\nu}\right)^{t-1} \mu(H \beta-\beta)
$$

Theorem 12. Suppose $M$ is a concave Orlicz function, and $H:\left(\ell_{M}(\lambda)\right)_{\mu} \longrightarrow\left(\ell_{M}(\lambda)\right)_{\mu}$ is Kannan $\mu$-contraction mapping, where $\mu(\beta)=\sum_{y=0}^{\infty} \lambda_{y} M\left(\left|\beta_{y}\right|\right)$, for all $\beta \in \ell_{M}(\lambda)$; hence, $H$ has a unique fixed point.

Proof. It is easy so omitted.

Definition 13. Assume $\left(\ell_{M}(\lambda)\right)_{\mu}$ is a pr-quasi normed (sss), $H:\left(\ell_{M}(\lambda)\right)_{\mu} \longrightarrow\left(\ell_{M}(\lambda)\right)_{\mu}$ and $\zeta \in\left(\ell_{M}(\lambda)\right)_{\mu}$. The operator $H$ is called $\mu$-sequentially continuous at $\zeta$, if and only if, when $\lim _{y \longrightarrow \infty} \mu\left(\beta_{y}-\zeta\right)=0$, then $\lim _{y \longrightarrow \infty} \mu\left(H \beta_{y}-H \zeta\right)=0$.

Theorem 14. Let $M$ be a strictly convex Orlicz function satisfying $\Delta_{2}$-condition, and $H:\left(\ell_{M}(\lambda)\right)_{\mu} \longrightarrow\left(\ell_{M}(\lambda)\right)_{\mu}$, where $\mu(\beta)=\sum_{y=0}^{\infty} \lambda_{y} M\left(\left|\beta_{y}\right|\right)$, for every $\beta \in \ell_{M}(\lambda)$. The element $\eta \in\left(\ell_{M}(\lambda)\right)_{\mu}$ is the unique fixed point of $H$, if the next conditions are satisfied:

(i) $H$ is Kannan $\mu$-contraction mapping, (ii) $H$ is $\mu$-sequentially continuous at a point $\eta \in\left(\ell_{M}(\lambda)\right)_{\mu}$

(iii) There exists $\beta \in\left(\ell_{M}(\lambda)\right)_{\mu}$ such that the sequence of iterates $\left\{H^{t} \beta\right\}$ has a subsequence $\left\{H^{t} p \beta\right.$ converging to $\eta$.

Proof. Since $M$ is a strictly convex Orlicz function satisfying $\Delta_{2}$-condition, then there exists $k>2$ such that $2 M(u)<M(2 u)<k M(u)$, for all $u \geq 0$. Let the conditions be verified. If $\eta$ is not a fixed point of $H$, then $H \eta \neq \eta$. By the conditions (ii) and (iii), we have

$$
\begin{aligned}
\lim _{t_{p} \longrightarrow \infty} \mu\left(H^{t} p-\eta\right) & =0, \\
\lim _{t_{p} \longrightarrow \infty} \mu\left(H^{t_{p}+1} \beta-H \eta\right) & =0 .
\end{aligned}
$$

As the operator $H$ is Kannan $\mu$-contraction, one can see 


$$
\begin{aligned}
0<\mu(H \eta-\eta) & =\mu\left(\left(H \eta-H^{t_{p}+1} \beta\right)+\left(H^{t_{p}} \beta-\eta\right)+\left(H^{t_{p}+1} \beta-H^{t_{p}} \beta\right)\right) \\
& \leq \frac{k^{2}}{4} \mu\left(H^{t_{p}+1} \beta-H \eta\right)+\frac{k^{2}}{4} \mu\left(H^{t_{p}} \beta-\eta\right)+\frac{k}{2} \nu\left(\frac{v}{1-v}\right)^{t_{p}-1} \mu(H \beta-\beta) .
\end{aligned}
$$

Since $t_{p} \longrightarrow \infty$, this gives a contradiction. Hence, $\eta$ is a fixed point of $H$. To prove that the uniqueness of the fixed point $\eta$. For different fixed points $\eta, \zeta \in\left(\ell_{M}(\lambda)\right)_{\mu}$ of $H$. Therefore, one has

$$
\mu(\eta-\zeta) \leq \mu(H \eta-H \zeta) \leq \nu(\mu(H \eta-\eta)+\mu(H \zeta-\zeta))=0 .
$$

So, $\eta=\zeta$.

Example 15. Assume $H:\left(\ell_{M}(\lambda)\right)_{\mu} \longrightarrow\left(\ell_{M}(\lambda)\right)_{\mu}$, where $M(t)=\sqrt[3]{t}+\sqrt[4]{t}$ and $\mu(\beta)=\sum_{y=0}^{\infty} \lambda_{y} M\left(\left|\beta_{y}\right|\right)$, for all $\beta \in \ell_{M}(\lambda)$ and

$$
H(\beta)= \begin{cases}\frac{\beta}{18}, & \mu(\beta) \in[0,1), \\ \frac{\beta}{20}, & \mu(\beta) \in[1, \infty) .\end{cases}
$$

As for each $\beta_{1}, \beta_{2} \in\left(\ell_{M}(\lambda)\right)_{\mu}$ with $\mu\left(\beta_{1}\right), \mu\left(\beta_{2}\right) \in[0,1)$, one has

$$
\begin{aligned}
\mu\left(H \beta_{1}-H \beta_{2}\right) & =\mu\left(\frac{\beta_{1}}{18}-\frac{\beta_{2}}{18}\right) \leq \frac{1}{\sqrt[4]{17}}\left(\mu\left(\frac{17 \beta_{1}}{18}\right)+\mu\left(\frac{17 \beta_{2}}{18}\right)\right) \\
& =\frac{1}{\sqrt[4]{17}}\left(\mu\left(H \beta_{1}-\beta_{1}\right)+\mu\left(H \beta_{2}-\beta_{2}\right)\right) .
\end{aligned}
$$
has

For all $\beta_{1}, \beta_{2} \in\left(\ell_{M}(\lambda)\right)_{\mu}$ with $\mu\left(\beta_{1}\right), \mu\left(\beta_{2}\right) \in[1, \infty)$, one

$$
\begin{aligned}
\mu\left(H \beta_{1}-H \beta_{2}\right) & =\mu\left(\frac{\beta_{1}}{20}-\frac{\beta_{2}}{20}\right) \leq \frac{1}{\sqrt[4]{19}}\left(\mu\left(\frac{19 \beta_{1}}{20}\right)+\mu\left(\frac{19 \beta_{2}}{20}\right)\right) \\
& =\frac{1}{\sqrt[4]{19}}\left(\mu\left(H \beta_{1}-\beta_{1}\right)+\mu\left(H \beta_{2}-\beta_{2}\right)\right) .
\end{aligned}
$$

For all $\beta_{1}, \beta_{2} \in\left(\ell_{M}(\lambda)\right)_{\mu}$ with $\mu\left(\beta_{1}\right) \in[0,1)$ and $\mu\left(\beta_{2}\right) \in[1, \infty)$, we obtain

$$
\begin{aligned}
\mu\left(H \beta_{1}-H \beta_{2}\right) & =\mu\left(\frac{\beta_{1}}{18}-\frac{\beta_{2}}{20}\right) \leq \frac{1}{\sqrt[4]{17}} \mu\left(\frac{17 \beta_{1}}{18}\right)+\frac{1}{\sqrt[4]{19}} \mu\left(\frac{19 \beta_{2}}{20}\right) \\
& \leq \frac{1}{\sqrt[4]{17}}\left(\mu\left(\frac{17 \beta_{1}}{18}\right)+\mu\left(\frac{19 \beta_{2}}{20}\right)\right) \\
& =\frac{1}{\sqrt[4]{17}}\left(\mu\left(H \beta_{1}-\beta_{1}\right)+\mu\left(H \beta_{2}-\beta_{2}\right)\right) .
\end{aligned}
$$

Hence, the operator $H$ is Kannan $\mu$-contraction. As $\mu$ verifies the Fatou property. From Theorem 11, the operator $H$ has a unique fixed point $\theta \in\left(\ell_{M}(\lambda)\right)_{\mu}$.
Assume $\left\{\beta^{(y)}\right\} \subseteq\left(\ell_{M}(\lambda)\right)_{\mu}$ is such that $\lim _{y \rightarrow \infty} \mu$ $\left(\beta^{(y)}-\beta^{(0)}\right)=0$, where

$\beta^{(0)} \in\left(\ell_{M}(\lambda)\right)_{\mu}$ with $\mu\left(\beta^{(0)}\right)=1$.

As the prequasi norm $\mu$ is continuous, one can see

$$
\begin{aligned}
\lim _{t_{p} \longrightarrow \infty} \mu\left(H \beta^{(y)}-H \beta^{(0)}\right) & =\lim _{t_{p} \longrightarrow \infty} \mu\left(\frac{\beta^{(y)}}{18}-\frac{\beta^{(0)}}{20}\right) \\
& =\mu\left(\frac{\beta^{(0)}}{180}\right)>0 .
\end{aligned}
$$

Therefore, $H$ is not $\mu$-sequentially continuous at $\beta^{(0)}$. Hence, the operator $H$ is not continuous at $\beta^{(0)}$.

Let $\mu(\beta)=\left[\sum_{y=0}^{\infty} \lambda_{y} M\left(\left|\beta_{y}\right|\right)\right]^{4}$, for all $\beta \in \ell_{M}(\lambda)$.

As for all $\beta_{1}, \beta_{2} \in\left(\ell_{M}(\lambda)\right)_{\mu}$ with $\mu\left(\beta_{1}\right), \mu\left(\beta_{2}\right) \in[0,1)$, one has

$$
\begin{aligned}
\mu\left(H \beta_{1}-H \beta_{2}\right) & =\mu\left(\frac{\beta_{1}}{18}-\frac{\beta_{2}}{18}\right) \leq \frac{8}{17}\left(\mu\left(\frac{17 \beta_{1}}{18}\right)+\mu\left(\frac{17 \beta_{2}}{18}\right)\right) \\
& =\frac{8}{17}\left(\mu\left(H \beta_{1}-\beta_{1}\right)+\mu\left(H \beta_{2}-\beta_{2}\right)\right) .
\end{aligned}
$$

For all $\beta_{1}, \beta_{2} \in\left(\ell_{M}(\lambda)\right)_{\mu}$ with $\mu\left(\beta_{1}\right), \mu\left(\beta_{2}\right) \in[1, \infty)$, one has

$$
\begin{aligned}
\mu\left(H \beta_{1}-H \beta_{2}\right) & =\mu\left(\frac{\beta_{1}}{20}-\frac{\beta_{2}}{20}\right) \leq \frac{8}{19}\left(\mu\left(\frac{19 \beta_{1}}{20}\right)+\mu\left(\frac{19 \beta_{2}}{20}\right)\right) \\
& =\frac{8}{19}\left(\mu\left(H \beta_{1}-\beta_{1}\right)+\mu\left(H \beta_{2}-\beta_{2}\right)\right) .
\end{aligned}
$$

For all $\beta_{1}, \beta_{2} \in\left(\ell_{M}(\lambda)\right)_{\mu}$ with $\mu\left(\beta_{1}\right) \in[0,1)$ and $\mu\left(\beta_{2}\right) \in[1, \infty)$, we get

$$
\begin{aligned}
\mu\left(H \beta_{1}-H \beta_{2}\right) & =\mu\left(\frac{\beta_{1}}{18}-\frac{\beta_{2}}{20}\right) \leq \frac{8}{17} \mu\left(\frac{17 \beta_{1}}{18}\right)+\frac{8}{19} \mu\left(\frac{19 \beta_{2}}{20}\right) \\
& \leq \frac{8}{17}\left(\mu\left(\frac{17 \beta_{1}}{18}\right)+\mu\left(\frac{19 \beta_{2}}{20}\right)\right) \\
& =\frac{8}{17}\left(\mu\left(H \beta_{1}-\beta_{1}\right)+\mu\left(H \beta_{2}-\beta_{2}\right)\right) .
\end{aligned}
$$

So, the operator $H$ is Kannan $\mu$-contraction and $H^{t}(\beta)=$ $\begin{cases}\beta / 18^{t} & \mu(\beta) \in[0,1)\end{cases}$ $\begin{cases}\beta / 20^{t} & \mu(\beta) \in[1, \infty)\end{cases}$

Clearly, $H$ is $\mu$-sequentially continuous at $\theta \in\left(\ell_{M}(\lambda)\right)_{\mu}$ and $\left\{H^{t} \beta\right\}$ contains a subsequence $\left\{H^{t} p \beta\right.$ converging to $\theta$. From Theorem 14, then $\theta \in\left(\ell_{M}(\lambda)\right)_{\mu}$ is the unique fixed point of $H$. 
Example 5. Assume $H:\left(\ell_{M}(\lambda)\right)_{\mu} \longrightarrow\left(\ell_{M}(\lambda)\right)_{\mu}$ where $M(t)=t^{2}$ and $\mu(\beta)=\sqrt{\sum_{y=0}^{\infty} \lambda_{y} M\left(\left|\beta_{y}\right|\right)}$, for all $\beta \in \ell_{M}(\lambda)$ and

$$
H(\beta)= \begin{cases}\frac{\beta}{4}, & \mu(\beta) \in[0,1), \\ \frac{\beta}{5}, & \mu(\beta) \in[1, \infty) .\end{cases}
$$

As for each $\beta_{1}, \beta_{2} \in\left(\ell_{M}(\lambda)\right)_{\mu}$ with $\mu\left(\beta_{1}\right), \mu\left(\beta_{2}\right) \in[0,1)$, one has

$$
\begin{aligned}
\mu\left(H \beta_{1}-H \beta_{2}\right) & =\mu\left(\frac{\beta_{1}}{4}-\frac{\beta_{2}}{4}\right) \leq \frac{1}{3}\left(\mu\left(\frac{3 \beta_{1}}{4}\right)+\mu\left(\frac{3 \beta_{2}}{4}\right)\right) \\
& =\frac{1}{3}\left(\mu\left(H \beta_{1}-\beta_{1}\right)+\mu\left(H \beta_{2}-\beta_{2}\right)\right) .
\end{aligned}
$$
has

For all $\beta_{1}, \beta_{2} \in\left(\ell_{M}(\lambda)\right)_{\mu}$ with $\mu\left(\beta_{1}\right), \mu\left(\beta_{2}\right) \in[1, \infty)$, one

$$
\begin{aligned}
\mu\left(H \beta_{1}-H \beta_{2}\right) & =\mu\left(\frac{\beta_{1}}{5}-\frac{\beta_{2}}{5}\right) \leq \frac{1}{4}\left(\mu\left(\frac{4 \beta_{1}}{5}\right)+\mu\left(\frac{4 \beta_{2}}{5}\right)\right) \\
& =\frac{1}{4}\left(\mu\left(H \beta_{1}-\beta_{1}\right)+\mu\left(H \beta_{2}-\beta_{2}\right)\right) .
\end{aligned}
$$

For all $\beta_{1}, \beta_{2} \in\left(\ell_{M}(\lambda)\right)_{\mu}$ with $\mu\left(\beta_{1}\right) \in[0,1)$ and $\mu\left(\beta_{2}\right) \in[1, \infty)$, we get

$$
\begin{aligned}
\mu\left(H \beta_{1}-H \beta_{2}\right) & =\mu\left(\frac{\beta_{1}}{4}-\frac{\beta_{2}}{5}\right) \leq \frac{1}{3} \mu\left(\frac{3 \beta_{1}}{4}\right)+\frac{1}{4} \mu\left(\frac{4 \beta_{2}}{5}\right) \\
& \leq \frac{1}{3}\left(\mu\left(\frac{3 \beta_{1}}{4}\right)+\mu\left(\frac{4 \beta_{2}}{5}\right)\right) \\
& =\frac{1}{3}\left(\mu\left(H \beta_{1}-\beta_{1}\right)+\mu\left(H \beta_{2}-\beta_{2}\right)\right) .
\end{aligned}
$$

Hence, the operator $H$ is Kannan $\mu$-contraction. As $\mu$ satisfies the Fatou property. From Theorem 11, the operator $H$ has one fixed point $\theta \in\left(\ell_{M}(\lambda)\right)_{\mu}$.

Suppose $\quad\left\{\beta^{(y)}\right\} \subseteq\left(\ell_{M}(\lambda)\right)_{\mu} \quad$ is so that $\lim _{y \rightarrow \infty} \mu\left(\beta^{(y)}-\beta^{(0)}\right)=0$, where.

$\beta^{(0)} \in\left(\ell_{M}(\lambda)\right)_{\mu}$ with $\mu\left(\beta^{(0)}\right)=1$. As the prequasi norm $\mu$ is continuous, one can see

$$
\begin{aligned}
\lim _{y \longrightarrow \infty} \mu\left(H \beta^{(y)}-H \beta^{(0)}\right) & =\lim _{y \longrightarrow \infty} \mu\left(\frac{\beta^{(y)}}{4}-\frac{\beta^{(0)}}{5}\right) \\
& =\mu\left(\frac{\beta^{(0)}}{20}\right)>0 .
\end{aligned}
$$

Therefore, $H$ is not $\mu$-sequentially continuous at $\beta^{(0)}$. Hence, the map $H$ is not continuous at $\beta^{(0)}$.

Let $\mu(\beta)=\sum_{y=0}^{\infty} \lambda_{y} M\left(\left|\beta_{y}\right|\right)$, for every $\beta \in \ell_{M}(\lambda)$.

As for each $\beta_{1}, \beta_{2} \in\left(\ell_{M}(\lambda)\right)_{\mu}$ with $\mu\left(\beta_{1}\right), \mu\left(\beta_{2}\right) \in[0,1)$, one has

$$
\begin{aligned}
\mu\left(H \beta_{1}-H \beta_{2}\right) & =\mu\left(\frac{\beta_{1}}{4}-\frac{\beta_{2}}{4}\right) \leq \frac{2}{9}\left(\mu\left(\frac{3 \beta_{1}}{4}\right)+\mu\left(\frac{3 \beta_{2}}{4}\right)\right) \\
& =\frac{2}{9}\left(\mu\left(H \beta_{1}-\beta_{1}\right)+\mu\left(H \beta_{2}-\beta_{2}\right)\right) .
\end{aligned}
$$

For all $\beta_{1}, \beta_{2} \in\left(\ell_{M}(\lambda)\right)_{\mu}$ with $\mu\left(\beta_{1}\right), \mu\left(\beta_{2}\right) \in[1, \infty)$, one has

$$
\begin{aligned}
\mu\left(H \beta_{1}-H \beta_{2}\right) & =\mu\left(\frac{\beta_{1}}{5}-\frac{\beta_{2}}{5}\right) \leq \frac{1}{8}\left(\mu\left(\frac{4 \beta_{1}}{5}\right)+\mu\left(\frac{4 \beta_{2}}{5}\right)\right) \\
& =\frac{1}{8}\left(\mu\left(H \beta_{1}-\beta_{1}\right)+\mu\left(H \beta_{2}-\beta_{2}\right)\right) .
\end{aligned}
$$

For all $\beta_{1}, \beta_{2} \in\left(\ell_{M}(\lambda)\right)_{\mu}$ with $\mu\left(\beta_{1}\right) \in[0,1)$ and $\mu\left(\beta_{2}\right) \in[1, \infty)$, we obtain

$$
\begin{aligned}
\mu\left(H \beta_{1}-H \beta_{2}\right) & =\mu\left(\frac{\beta_{1}}{4}-\frac{\beta_{2}}{5}\right) \leq \frac{2}{9} \mu\left(\frac{3 \beta_{1}}{4}\right)+\frac{1}{8} \mu\left(\frac{4 \beta_{2}}{5}\right) \\
& \leq \frac{2}{9}\left(\mu\left(\frac{3 \beta_{1}}{4}\right)+\mu\left(\frac{4 \beta_{2}}{5}\right)\right) \\
& =\frac{2}{9}\left(\mu\left(H \beta_{1}-\beta_{1}\right)+\mu\left(H \beta_{2}-\beta_{2}\right)\right) .
\end{aligned}
$$

So, the operator $H$ is Kannan $\mu$-contraction and. $H^{t}(\beta)= \begin{cases}\beta / 4^{t} & \mu(\beta) \in[0,1) \\ \beta / 5^{t} & \mu(\beta) \in[1, \infty)\end{cases}$

Obviously, $H$ is $\mu$-sequentially continuous at $\theta \in\left(\ell_{M}(\lambda)\right)_{\mu}$ and $\left\{H^{t} \beta\right\}$ has a subsequence $\left\{H^{t} p \beta\right\}$ converging to $\theta$. From Theorem 14 , then $\theta \in\left(\ell_{M}(\lambda)\right)_{\mu}$ is the unique fixed point of $H$.

Example 16. Suppose $H:\left(\ell_{M}(\lambda)\right)_{\mu} \longrightarrow\left(\ell_{M}(\lambda)\right)_{\mu}$, where $M(t)=\sqrt[3]{t}+\sqrt[4]{t}$ and $\mu(\beta)=\left(\sum_{y=0}^{\infty} \lambda_{y} M\left(\left|\beta_{y}\right|\right)\right)^{4}$, for every $\beta \in \ell_{M}(\lambda)$ and

$$
H(\beta)= \begin{cases}\frac{1}{18}\left(e_{0}+\beta\right), & \beta_{0} \in\left(-\infty, \frac{1}{17}\right), \\ \frac{1}{17} e_{0}, & \beta_{0}=\frac{1}{17}, \\ \frac{1}{18} e_{0}, & \beta_{0} \in\left(\frac{1}{17}, \infty\right) .\end{cases}
$$

As for each $\beta, \eta \in\left(\ell_{M}(\lambda)\right)_{\mu}$ with $\beta_{0}, \eta_{0} \in(-\infty, 1 / 17)$, one has

$$
\begin{aligned}
\mu(H \beta-H \eta) & =\mu\left(\frac{1}{18}\left(\beta_{0}-\eta_{0}, \beta_{1}-\eta_{1}, \beta_{2}-\eta_{2}, \ldots\right)\right) \\
& \leq \frac{8}{17}\left(\mu\left(\frac{17 \beta}{18}\right)+\mu\left(\frac{17 t}{18}\right)\right) \\
& \leq \frac{8}{17}(\mu(H \beta-\beta)+\mu(H \eta-\eta)) .
\end{aligned}
$$


For every $\beta, \eta \in\left(\ell_{M}(\lambda)\right)_{\mu}$ with $\beta_{0}, \eta_{0} \in(1 / 17, \infty)$, then for all $\epsilon>0$ one has

$$
\mu(H \beta-H \eta)=0 \leq \epsilon(\mu(H \beta-\beta)+\mu(H \eta-\eta)) .
$$

For every $\beta, \eta \in\left(\ell_{M}(\lambda)\right)_{\mu}$ with $\beta_{0} \in(-\infty, 1 / 17)$ and $\eta_{0} \in(1 / 17, \infty)$, we get

$$
\begin{aligned}
\mu(H \beta-H \eta) & =\mu\left(\frac{\beta}{18}\right) \leq \frac{1}{17} \mu\left(\frac{17 \beta}{18}\right)=\frac{1}{17} \mu(H \beta-\beta) \\
& \leq \frac{1}{17}(\mu(H \beta-\beta)+\mu(H \eta-\eta)) .
\end{aligned}
$$

Hence, the operator $H$ is Kannan $\mu$-contraction. Evidently, $H$ is $\mu$-sequentially continuous at $1 / 17 e_{0} \in\left(\ell_{M}(\lambda)\right)_{\mu}$, and we have $\beta \in\left(\ell_{M}(\lambda)\right)_{\mu}$ with $\beta_{0} \in(-\infty, 1 / 17)$ under $\left\{H^{t} \beta\right\}=\left\{\sum_{n=1}^{t} 1 / 18^{n} e_{0}+1 / 18^{t} \beta\right\}$ contains a subsequence $\left\{H^{t} p \beta=\left\{\sum_{n=1}^{t_{p}} 1 / 18^{n} e_{0}+1 / 18^{t_{p}} \beta\right\}\right.$ converging to $1 / 17 e_{0}$. From Theorem 14, the map $H$ has a unique fixed point $1 / 17 e_{0} \in\left(\ell_{M}(\lambda)\right)_{\mu}$. Observe that $H$ is not continuous at $1 / 17 e_{0} \in\left(\ell_{M}(\lambda)\right)_{\mu}$.

If $\mu(\beta)=\sum_{y \in \mathscr{L}^{+}} \lambda_{y} M\left(\left|\beta_{y}\right|\right)$, for every $\beta \in \ell_{M}(\lambda)$. As for all $\beta, \eta \in\left(\ell_{M}(\lambda)\right)_{\mu}$ with $\beta_{0}, \eta_{0} \in(-\infty, 1 / 17)$, one has

$$
\begin{aligned}
\mu(H \beta-H \eta) & =\mu\left(\frac{1}{18}\left(\beta_{0}-\eta_{0}, \beta_{1}-\eta_{1}, \beta_{2}-\eta_{2}, \ldots\right)\right) \\
& \leq \frac{1}{\sqrt[4]{17}}\left(\mu\left(\frac{17 \beta}{18}\right)+\mu\left(\frac{17 t}{18}\right)\right) \\
& \leq \frac{1}{\sqrt[4]{17}}(\mu(H \beta-\beta)+\mu(H \eta-\eta)) .
\end{aligned}
$$

For each $\beta, \eta \in\left(\ell_{M}(\lambda)\right)_{\mu}$ with $\beta_{0}, \eta_{0} \in(1 / 17, \infty)$, then for all $\epsilon>0$ we get

$$
\mu(H \beta-H \eta)=0 \leq \epsilon(\mu(H \beta-\beta)+\mu(H \eta-\eta)) .
$$

For every $\beta, \eta \in\left(\ell_{M}(\lambda)\right)_{\mu}$ with $\beta_{0} \in(-\infty, 1 / 17)$ and $\eta_{0} \in(1 / 17, \infty)$, this gives

$$
\begin{aligned}
\mu(H \beta-H \eta) & =\mu\left(\frac{\beta}{18}\right) \leq \frac{1}{\sqrt[4]{17}} \mu\left(\frac{17 \beta}{18}\right)=\frac{1}{\sqrt[4]{17}} \mu(H \beta-\beta) \\
& \leq \frac{1}{\sqrt[4]{17}}(\mu(H \beta-\beta)+\mu(H \eta-\eta))
\end{aligned}
$$

So, the operator $H$ is Kannan $\mu$-contraction. As $\mu$ satisfies the Fatou property. From Theorem 11, the operator $H$ holds one fixed point $1 / 17 e_{0} \in\left(\ell_{M}(\lambda)\right)_{\mu}$.

Example 6. Assume $H:\left(\ell_{M}(\lambda)\right)_{\mu} \longrightarrow\left(\ell_{M}(\lambda)\right)_{\mu}$, where $M(t)=t^{2}+2 t$ and $\mu(\beta)=\sum_{y=0}^{\infty} \lambda_{y} M\left(\left|\beta_{y}\right|\right)$, for every $\beta \in \ell_{M}(\lambda)$ and

$$
H(\beta)= \begin{cases}\frac{1}{6}\left(e_{1}+\beta\right), & \beta_{0} \in\left(-\infty, \frac{1}{5}\right), \\ \frac{1}{5} e_{1}, & \beta_{0}=\frac{1}{5}, \\ \frac{1}{6} e_{1}, & \beta_{0} \in\left(\frac{1}{5}, \infty\right) .\end{cases}
$$
has

As for each $\beta, \eta \in\left(\ell_{M}(\lambda)\right)_{\mu}$ with $\beta_{0}, \eta_{0} \in(-\infty, 1 / 5)$, one

$$
\begin{aligned}
\mu(H \beta-H \eta) & =\mu\left(\frac{1}{6}\left(\beta_{0}-\eta_{0}, \beta_{1}-\eta_{1}, \beta_{2}-\eta_{2}, \ldots\right)\right) \\
& \leq \frac{2}{5}\left(\mu\left(\frac{5 \beta}{6}\right)+\mu\left(\frac{5 t}{6}\right)\right) \\
& \leq \frac{2}{5}(\mu(H \beta-\beta)+\mu(H v-\eta)) .
\end{aligned}
$$

Suppose $\beta, \eta \in\left(\ell_{M}(\lambda)\right)_{\mu}$ with $\beta_{0}, \eta_{0} \in(1 / 5, \infty)$, then for any $\epsilon>0$ we obtain

$$
\mu(H \beta-H \eta)=0 \leq \epsilon(\mu(H \beta-\beta)+\mu(H \eta-\eta)) .
$$

Assume $\beta, \eta \in\left(\ell_{M}(\lambda)\right)_{\mu}$ with $\beta_{0} \in(-\infty, 1 / 5)$ and $\eta_{0} \in(1 / 5, \infty)$, one can see

$$
\begin{aligned}
\mu(H \beta-H \eta) & =\mu\left(\frac{\beta}{6}\right) \leq \frac{1}{5} \mu\left(\frac{5 \beta}{6}\right)=\frac{1}{5} \mu(H \beta-\beta) \\
& \leq \frac{1}{5}(\mu(H \beta-\beta)+\mu(H \eta-\eta)) .
\end{aligned}
$$

Hence, the operator $H$ is Kannan $\mu$-contraction. Clearly, $H$ is $\mu$-sequentially continuous at $1 / 5 e_{1} \in\left(\ell_{M}(\lambda)\right)_{\mu}$ and there exists $\beta \in\left(\ell_{M}(\lambda)\right)_{\mu}$ with $\beta_{0} \in(-\infty, 1 / 5)$ under $\left\{H^{t} \beta\right\}=\left\{\sum_{n=1}^{n=1} 1 / 6^{n} e_{1}+1 / 6^{t} \beta\right\}$ contains a subsequence $\left\{H^{t_{p}} \beta\right\}=\left\{\sum_{n=1}^{t_{p}} 1 / 6^{n} e_{1}+1 / 6^{t_{p}} \beta\right\}$ converging to $1 / 5 e_{1}$. From Theorem 14, the operator $H$ holds a unique fixed point $1 / 5 e_{1} \in\left(\ell_{M}(\lambda)\right)_{\mu}$. Observe that $H$ is not continuous at $1 / 5 e_{1} \in\left(\ell_{M}(\lambda)\right)_{\mu}$.

If $M(t)=t^{2}$ and $\mu(\beta)=\sqrt{\sum_{y=0}^{\infty} \lambda_{y} M\left(\left|\beta_{y}\right|\right)}$, for every $\beta \in \ell_{M}(\lambda)$.

Since for all $\beta, \eta \in\left(\ell_{M}(\lambda)\right)_{\mu}$ with $\beta_{0}, \eta_{0} \in(-\infty, 1 / 5)$, one has

$$
\begin{aligned}
\mu(H \beta-H \eta) & =\mu\left(\frac{1}{6}\left(\beta_{0}-\eta_{0}, \beta_{1}-\eta_{1}, \beta_{2}-\eta_{2}, \ldots\right)\right) \\
& \leq \frac{1}{5}\left(\mu\left(\frac{5 \beta}{6}\right)+\mu\left(\frac{5 \eta}{6}\right)\right) \\
& \leq \frac{1}{5}(\mu(H \beta-\beta)+\mu(H \eta-\eta)) .
\end{aligned}
$$

If $\beta, \eta \in\left(\ell_{M}(\lambda)\right)_{\mu}$ with $\beta_{0}, \eta_{0} \in(1 / 5, \infty)$, then for all $\epsilon>0$ one has 
$\mu(H \beta-H \eta)=0 \leq \epsilon(\mu(H \beta-\beta)+\mu(H \eta-\eta))$.

Assume $\beta, \eta \in\left(\ell_{M}(\lambda)\right)_{\mu}$ with $\beta_{0} \in(-\infty, 1 / 5)$ and $\eta_{0} \in(1 / 5, \infty)$, we get

$$
\begin{aligned}
\mu(H \beta-H \eta) & =\mu\left(\frac{\beta}{6}\right) \leq \frac{1}{5} \mu\left(\frac{5 \beta}{6}\right)=\frac{1}{5} \mu(H \beta-\beta) \\
& \leq \frac{1}{5}(\mu(H \beta-\beta)+\mu(H \eta-\eta)) .
\end{aligned}
$$

So, the operator $H$ is Kannan $\mu$-contraction. As $\mu$ satisfies the Fatou property. From Theorem 11, the operator $H$ contains one fixed point $1 / 5 e_{1} \in\left(\ell_{M}(\lambda)\right)_{\mu}$.

\section{Kannan Nonexpansive Operator}

We have presented in this section the uniform convexity of the space $\left(\ell_{M}(\lambda)\right)_{\mu}$, where

$$
\ell_{M}(\lambda)=\left\{u \in \mathbf{R}^{\mathscr{X}^{+}}: \varrho(\omega u)<\infty \text {, for some } \omega>0\right\},
$$

and $\varrho(u)=\sum_{y=0}^{\infty} \lambda_{y} M\left(\left|u_{y}\right|\right)$, under the Luxemburg norm

$$
\mu(u)=\inf \left\{\omega>0: \varrho\left(\frac{u}{\omega}\right) \leq 1\right\} .
$$

\section{Definition 12.}

(1) The continuous function $M$ is called strictly convex (SC), if

$$
M\left(\frac{v+t}{2}\right)<\frac{M(v)+M(t)}{2},
$$

for all $v, t \in[0, \infty)$ and $v \neq t$.

(2) [24] The following statements are equivalent:

(i) $M$ is a uniformly convex function on $[0, \infty)$.

(ii) For any $\epsilon>0$ and $u_{0}>0$, there exists a number $\delta \in(0,1)$ such that for all $u, v$, and $|u-v| \geq \epsilon \max \{|u|,|v|\} \geq \epsilon u_{0}$ imply

$$
M\left(\frac{u+v}{2}\right) \leq \frac{1-\delta}{2}(M(u)+M(v))
$$

$$
\text { if } u \geq u_{0} \text {. }
$$

(iii) For any $u_{0}>0$ and $a \in(0,1)$, there exists a number $\delta \in(0,1)$ such that

if $u \geq u_{0}$.

(3) [25] A normed space $(X, \mu)$ is said to be strictly convex if for any $u, v \in X$ and $b>0$ satisfying $\mu(u) \leq b, \quad \mu(v) \leq b, \quad$ and $\mu(u-v)>0 \quad$ imply $\mu(u+v / 2)<b$.

(4) [26] A normed space $(X, \mu)$ is said to be uniformly convex if for any $b>0$ and $\epsilon>0$, there exists $\delta>0$ such that for all $u, v \in X$ satisfying $\mu(u) \leq b, \mu(v) \leq b$ and $\mu(u-v) \geq \epsilon$ imply $\mu(u+v / 2) \leq b-\delta$.

Theorem 17. If $\lim _{n \longrightarrow \infty} \mu\left(x_{n}\right)=b, \lim _{n \longrightarrow \infty} \mu\left(y_{n}\right)=b$ and $\lim _{n \longrightarrow \infty} \mu\left(x_{n}+y_{n} / 2\right)=b$ imply $\lim _{n \longrightarrow \infty} \mu\left(x_{n}-y_{n}\right)=0$, for all $\left\{x_{n}\right\},\left\{y_{n}\right\} \subset \ell_{M}(\lambda)$ and $b>0$, then $\ell_{M}(\lambda)$ is uniformly convex, where $M$ is a convex Orlicz function satisfying $\Delta_{2}$-condition.

Proof. Let the conditions be satisfied and $\ell_{M}(\lambda)$ is not uniformly convex, then there exists $\epsilon_{0}>0$ and $\left\{x_{n}\right\},\left\{y_{n}\right\} \subset \ell_{M}(\lambda)$ such that $\mu\left(x_{n}\right) \leq b, \mu\left(y_{n}\right) \leq b, \mu\left(x_{n}-\right.$ $\left.y_{n}\right) \geq \epsilon_{0}$ we get $\mu\left(x_{n}+y_{n} / 2\right)>b-1 / n$, for some $b>0$. To prove that $\lim _{n \longrightarrow \infty} \mu\left(x_{n}\right)=b$, let $\lim _{n \longrightarrow \infty} \mu\left(x_{n}\right)=b_{1}<b$ and $\lim _{n \longrightarrow \infty} \mu\left(y_{n}\right)=b$. Since $M$ is satisfying $\Delta_{2}$-condition, we have $\lim _{n \longrightarrow \infty} \varrho\left(x_{n} / b_{1}\right)=1$ and $\lim _{n \longrightarrow \infty} \varrho\left(x_{n} / b\right)=1$. Hence,

$$
\begin{aligned}
\lim _{n \rightarrow \infty} \varrho\left(\frac{x_{n}+y_{n}}{2 b}\right) \leq & \frac{b_{1}}{2 b} \lim _{n \longrightarrow \infty} \varrho\left(\frac{x_{n}}{b_{1}}\right) \\
& \left.+\frac{1}{2} \lim _{n \longrightarrow \infty} \varrho\left(\frac{y_{n}}{b}\right)\right)<1 .
\end{aligned}
$$

This is equivalent to $\varlimsup_{n \rightarrow \infty} \mu\left(x_{n}+y_{n} / 2\right)<b$. This contradicts $\quad \lim _{n \rightarrow \infty} \mu\left(x_{n}+y_{n} / 2\right)>b, \quad$ so $\lim _{n \rightarrow \infty} \varrho\left(x_{n}\right)=b$. Similarly, we can prove that $\lim _{n \longrightarrow \infty} \varrho\left(y_{n}\right) b$. Also since

$$
1<\varliminf_{n \longrightarrow \infty} \varrho\left(\frac{x_{n}+y_{n}}{2 b}\right) \leq \lim _{n \longrightarrow \infty} \varrho\left(\frac{x_{n}+y_{n}}{2 b}\right) \leq \varlimsup_{n \longrightarrow \infty} \varrho\left(\frac{x_{n}+y_{n}}{2 b}\right) \leq \frac{1}{2}\left(\varlimsup_{n \longrightarrow \infty} \varrho\left(\frac{x_{n}}{b}\right)+\varlimsup_{n \longrightarrow \infty} \varrho\left(\frac{y_{n}}{b}\right)\right)=1 .
$$

Then $\quad \lim _{n \longrightarrow \infty} \varrho\left(x_{n}+y_{n} / 2 b\right)=1$. This implies $\lim _{n \longrightarrow \infty} \mu\left(x_{n}+y_{n} / 2\right)=b$. But $\lim _{n \longrightarrow \infty} \mu\left(x_{n}-y_{n}\right) \geq \epsilon_{0}>0$, this gives a contradiction.

Theorem 18. The space $\ell_{M}(\lambda)$ is uniformly convex, if $M$ is a uniformly convex Orlicz function satisfying $\Delta_{2}$-condition.
Proof. Assume the settings are satisfied, $\lim _{n \rightarrow \infty} \mu\left(x_{n}\right)=b$, $\lim _{n \longrightarrow \infty} \mu\left(y_{n}\right)=b$, and $\lim _{n \longrightarrow \infty} \mu\left(x_{n}+y_{n} / 2\right)=b$, we will prove that $\lim _{n \rightarrow \infty} \mu\left(x_{n}-y_{n}\right)=0$. For any $\epsilon \in(0,1 / 2)$, let us choose $u_{0}>0$ such that $M\left(2 u_{0}\right)<\epsilon$. Since $M$ is uniformly convex, then there exists $\delta \in(0,1)$ such that $|u-v| \geq \epsilon \max \{|u|,|v|\} \geq \epsilon u_{0}$ imply 


$$
M\left(\frac{u+v}{2}\right) \leq \frac{1-\delta}{2}(M(u)+M(v)) .
$$

For each $n \in \mathscr{Z}^{+}$, put

$$
\begin{aligned}
& G_{n}=\left\{i \in \mathscr{Z}^{+}:\left|\frac{x_{n}(i)}{b}\right|,\left|\frac{y_{n}(i)}{b}\right|<u_{0}\right\}, \\
& E_{n}=\left\{i \in \mathscr{Z}^{+}:\left|\frac{x_{n}(i)-y_{n}(i)}{b}\right|<\epsilon \max \left\{\left|\frac{x_{n}(i)}{b}\right|,\left|\frac{y_{n}(i)}{b}\right|\right\}<\epsilon u_{0}\right\}, \\
& F_{n}=\left\{i \in \mathscr{Z}^{+}:\left|\frac{x_{n}(i)-y_{n}(i)}{b}\right| \geq \epsilon \max \left\{\left|\frac{x_{n}(i)}{b}\right|,\left|\frac{y_{n}(i)}{b}\right|\right\} \geq \epsilon u_{0}\right\} .
\end{aligned}
$$

Then we deduce

$$
\sum_{i \in G_{n}} M\left(\left|\frac{x_{n}(i)-y_{n}(i)}{b}\right|\right) \leq M\left(2 u_{0}\right),
$$

and thus

$$
\begin{aligned}
\sum_{i \in E_{n}} M\left(\left|\frac{x_{n}(i)-y_{n}(i)}{b}\right|\right) & \leq 2 \epsilon \sum_{i \in \mathscr{Z}^{+}} M\left(\frac{\left|x_{n}(i)\right|+\left|y_{n}(i)\right|}{2 b}\right) \\
& \leq \epsilon\left(\sum_{i \in \mathscr{Z}^{+}} M\left(\left|\frac{x_{n}(i)}{b}\right|\right)+\sum_{i \in \mathscr{Z}^{+}} M\left(\left|\frac{y_{n}(i)}{b}\right|\right)\right) \leq 2 \epsilon .
\end{aligned}
$$

Hence, we get

$$
\begin{aligned}
0 & \leftarrow \frac{\mu\left(x_{n}\right)+\mu\left(y_{n}\right)}{2}-\mu\left(\frac{x_{n}+y_{n}}{2}\right) \\
= & \frac{\sum_{i \in \mathscr{Z}^{+}} M\left(\left|x_{n}(i) / b\right|\right)+\sum_{i \in \mathscr{Z}^{+}} M\left(y_{n}(i) / b\right)}{2} \\
& -\sum_{i \in \mathscr{Z}^{+}} M\left(\frac{\left|x_{n}(i)+y_{n}(i)\right|}{2 b}\right) \\
\geq & \frac{\sum_{i \in F_{n}} M\left(\left|x_{n}(i) / b\right|\right)+\sum_{i \in F_{n}} M\left(y_{n}(i) / b\right)}{2} \\
& -\sum_{i \in F_{n}} M\left(\frac{\left|x_{n}(i)+y_{n}(i)\right|}{2 b}\right) \\
\geq & \frac{\sum_{i \in F_{n}} M\left(\left|x_{n}(i) / b\right|\right)+\sum_{i \in F_{n}} M\left(y_{n}(i) / b\right)}{2} \\
& -\frac{1-\delta}{2}\left[\sum_{i \in F_{n}} M\left(\left|x_{n}(i) / b\right|\right)+\sum_{i \in F_{n}} M\left(y_{n}(i) / b\right)\right] \\
= & \frac{\delta}{2}\left[\sum_{i \in F_{n}} M\left(\left|x_{n}(i) / b\right|\right)+\sum_{i \in F_{n}} M\left(y_{n}(i) / b\right)\right] .
\end{aligned}
$$

Since $u_{0}$ and $\epsilon$ are arbitrary, then $\lim _{n \rightarrow \infty} \varrho\left(x_{n}-y_{n} / 2 b\right)=0$. As $M$ verifies $\Delta_{2}$-condition. Therefore, $\lim _{n \longrightarrow \infty} \mu\left(x_{n}-y_{n}\right)=0$. From Theorem 17, the proof follows.

Here, we discuss the property $(R)$ and the $\mu$-normal structure property of the space $\left(\ell_{M}(\lambda)\right)_{\mu}$.

Definition 13. The space $(Y)_{\mu}$ holds the property $(R)$, if for all decreasing sequence $\left\{\Phi_{x}\right\}_{x \in \mathscr{Z}^{+}}$of $\mu$-closed and $\mu$-convex nonempty subsets of $(Y)_{\mu}$ so that $\sup _{x \in \mathscr{Z}^{+}} d_{\mu}\left(\beta, \Phi_{x}\right)<\infty$, for some $\beta \in(Y)_{\mu}$; hence, we have $\cap_{x \in \mathscr{F}^{+}} \Phi_{x} \neq \varnothing$.

Definition 14. The space $(Y)_{\mu}$ holds the $\mu$-normal structure property if for all nonempty $\mu$-bounded, $\mu$-convex, and $\mu$-closed subset $\Phi$ of $(Y)_{\mu}$ not decreased to one point, there exists $\beta \in \Phi$ with

$\sup _{\eta \in \Phi} \mu(\beta-\eta)<v_{\mu}(\Phi):=\sup \{\mu(\beta-\eta): \beta, \eta \in \Phi\}<\infty$.

Theorem 19. If $M$ is a uniformly convex Orlicz function satisfying $\Delta_{2}$-condition, then

(1) Assume $\Phi$ is a nonempty $\mu$-closed and $\mu$-convex subset of $\left(\ell_{M}(\lambda)\right)_{\mu}$. For $\beta \in\left(\ell_{M}(\lambda)\right)_{\mu}$ with

$$
d_{\mu}(\beta, \Phi)=\inf \{\mu(\beta-\eta): \eta \in \Phi\}<\infty .
$$

Therefore, we have one $\phi \in \Phi$ with $d_{\mu}(\beta, \Phi)=\mu(\beta-\phi)$.

(2) $\left(\ell_{M}(\lambda)\right)_{\mu}$ satisfies the property $(R)$.

Proof. For (1), assume $\beta \notin \Phi$ as $\Phi$ is $\mu$-closed. So, one has $D:=d_{\mu}(\beta, \Phi)>0$. Therefore, there is $\eta_{t} \in \Phi$ so that $\lim _{t \rightarrow \infty} \mu\left(\beta-\eta_{t}\right)=D$. To prove that $\left\{\eta_{t}\right\}$ is a $\mu$-Cauchy. For any two subsequences $\left\{\eta_{t_{a}}\right\}$ and $\left\{\eta_{t_{b}}\right\} \subset\left\{\eta_{t}\right\}$, we have $\mu\left(\eta_{t_{a}}+\eta_{t_{b}} / 2-\beta\right) \geq D, \quad$ as $\quad \lim _{a \longrightarrow \infty} \mu\left(\beta-\eta_{t_{a}}\right)=D$ and $\lim _{b \longrightarrow \infty} \mu\left(\beta-\eta_{t_{b}}\right)=D$. Moreover,

$$
\mu\left(\frac{\eta_{t_{a}}+\eta_{t_{b}}}{2}-\beta\right)=\mu\left(\frac{\eta_{t_{a}}-\beta}{2}+\frac{\eta_{t_{b}}-\beta}{2}\right)<\frac{1}{2}(D+D)=D .
$$


Therefore, we have $\lim _{a, b \rightarrow \infty} \mu\left(\eta_{t_{a}}+\eta_{t_{b}} / 2-\beta\right)=D$. Since the space $\left(\ell_{M}(\lambda)\right)_{\mu}$ is uniformly convex, we get

$$
\lim _{a, b \longrightarrow \infty} \mu\left(\eta_{t_{a}}-\beta-\left(\eta_{t_{b}}-\beta\right)\right)=\lim _{a, b \longrightarrow \infty} \mu\left(\eta_{t_{a}}-\eta_{t_{b}}\right)=0
$$

Thus, $\left\{\eta_{t}\right\}$ is a $\mu$-Cauchy in $\Phi$. Since $\Phi$ is closed and the space $\left(\ell_{M}(\lambda)\right)_{\mu}$ is complete, then there exists $\phi \in \Phi$ with $\mu(\beta-\phi)=d_{\mu}(\beta, \Phi)$. Since the space $\left(\ell_{M}(\lambda)\right)_{\mu}$ is uniformly convex, then it is (SC), which implies the uniqueness of $\phi$. To show (2), for some $t_{0} \in \mathscr{Z}^{+}$, suppose $\beta \notin \Phi_{t_{0}}$. Since $\left(d_{\mu}\right.$ $\left.\left(\beta, \Phi_{t}\right)\right)_{t \in \mathscr{Z}^{+}} \in \ell_{\infty}$ is increasing. Set $\lim _{t \longrightarrow \infty} d_{\mu}\left(\beta, \Phi_{t}\right)=D$, when $D>0$. Otherwise, $\beta \in \Phi_{t}$, for each $t \in \mathscr{Z}^{+}$. From (1), we have a unique $\eta_{t} \in \Phi_{t}$ with $d_{\mu}\left(\beta, \Phi_{t}\right)=\mu\left(\beta-\eta_{t}\right)$, for all $t \in \mathscr{Z}^{+}$. A consistent proof will show that $\left\{\eta_{t} / 2\right\} \mu$-converges to some $\eta \in\left(\ell_{M}(\lambda)\right)_{\mu}$. Since $\left\{\Phi_{t}\right\}$ are $\mu$-convex, decreasing and $\mu$-closed, we get $2 \eta \in \cap_{t \in \mathscr{Z}^{+}} \Phi_{t}$.

Theorem 20. If $M$ is a uniformly convex Orlicz function satisfying $\Delta_{2}$-condition, then $\left(\ell_{M}(\lambda)\right)_{\mu}$ has the $\mu$-normal structure property.

Proof. Let the conditions are satisfied. Theorem 18 gives that $\left(\ell_{M}(\lambda)\right)_{\mu}$ is uniformly convex. Assume $\Phi$ is a $\mu$-bounded, $\mu$-convex, and $\mu$-closed subset of $\left(\ell_{M}(\lambda)\right)_{\mu}$ not decreased to one point. Hence, $v_{\mu}(\Phi)>0$. Set $D=v_{\mu}(\Phi)$. Let $\beta, \eta \in \Phi$ with $\beta \neq \eta$. Hence, $\mu(\beta-\eta / 2)>0$. For every $\phi \in \Phi$, one has $\mu(\beta-\phi) \leq D$ and $\mu(\eta-\phi) \leq D$. As $\Phi$ is $\mu$-convex, then $\beta+\eta / 2 \in \Phi$. Hence,

$$
\mu\left(\frac{\beta+\eta}{2}-\phi\right)=\mu\left(\frac{(\beta-\phi)+(\eta-\phi)}{2}\right)<D,
$$

for every $\phi \in \Phi$. So

$$
\sup _{\phi \in \Phi} \mu\left(\frac{\beta+\eta}{2}-\phi\right)<D=v_{\mu}(\Phi) .
$$

Lemma 3. Let the space $\left(\ell_{M}(\lambda)\right)_{\mu}$ verify the $(R)$ property and the $\mu$-quasi-normal property. Assume $\Phi$ is a nonempty $\mu$-bounded, $\mu$-convex, and $\mu$-closed subset of $\left(\ell_{M}(\lambda)\right)_{\mu}$. Suppose $H: \Phi \longrightarrow \Phi$ is a Kannan $\mu$-nonexpansive mapping. For $x>0$. If $W_{x}=\{\beta \in \Phi: \mu(\beta-H(\beta)) \leq x\} \neq \varnothing$. Set

$$
\Phi_{x}=\cap\left\{\mathscr{B}_{\mu}(t, v): H\left(W_{x}\right) \subset \mathscr{B}_{\mu}(t, v)\right\} \cap \Phi .
$$

Then $\Phi_{x}$ is a nonempty, $\mu$-convex, $\mu$-closed subset of $\Phi$ with $H\left(\Phi_{x}\right) \subset \Phi_{x} \subset W_{x}$ and $v_{\mu}\left(\Phi_{x}\right) \leq x$.

Proof. As $H\left(W_{x}\right) \subset \Phi_{x}$, this gives $\Phi_{x} \neq \varnothing$. Since the $\mu$-balls are $\mu$-convex, and $\mu$-closed, then $\Phi_{x}$ is a $\mu$-closed and $\mu$-convex subset of $\Phi$. To prove that $\Phi_{x} \subset W_{x}$. Assume $\beta \in \Phi_{x}$. If $\mu(\beta-H(\beta))=0$, we have $\beta \in W_{x}$. Otherwise, suppose $\mu(\beta-H(\beta))>0$. Set

$$
t=\sup \left\{\mu(H(\zeta)-H(\beta)): \zeta \in W_{x}\right\} .
$$

From the definition of $t$, then $H\left(W_{x}\right) \subset \mathscr{B}_{\mu}(H(\beta), t)$. Hence, $\Phi_{x} \subset \mathscr{B}_{\mu}(H(\beta), t)$, which implies $\mu(\beta-H(\beta)) \leq t$.
Assume $d>0$. Hence, there is $\zeta \in W_{x}$ so that $t-d \leq \mu(H(\zeta)-H(\beta))$. Then

$$
\begin{aligned}
\mu(\beta-H(\beta))-d & \leq t-d \leq \mu(H(\zeta)-H(\beta)) \\
& \leq \frac{1}{2}(\mu(\beta-H(\beta))+\mu(\zeta-H(\zeta))) \\
& \leq \frac{1}{2}(\mu(\beta-H(\beta))+x) .
\end{aligned}
$$

Since $d$ is arbitrarily positive, we have $\mu(\beta-H(\beta)) \leq x$, then we have $\beta \in W_{x}$. For $H\left(W_{x}\right) \subset \Phi_{x}$, we get $H\left(\Phi_{x}\right) \subset H\left(W_{x}\right) \subset \Phi_{x}$, this indicates $\Phi_{x}$ is $H$-invariant. Consequent to prove that $v_{\mu}\left(\Phi_{x}\right) \leq x$. As

$$
\mu(H(\beta)-H(\eta)) \leq \frac{1}{2}(\mu(\beta-H(\beta))+\mu(\eta-H(\eta))),
$$

For every $\beta, \eta \in W_{x}$. Let $\beta \in W_{x}$. So $H\left(W_{x}\right) \subset \mathscr{B}_{\mu}(H(\beta), x)$. From the definition of $\Phi_{x}$, one has $\Phi_{x} \subset \mathscr{B}_{\mu}(H(\beta), x)$. Hence, $H(\beta) \in \cap_{\eta \in \Phi_{x}} \mathscr{B}_{\mu}(\eta, x)$. Therefore, we have $\mu(\eta-\zeta) \leq x$, for every $\eta, \zeta \in \Phi_{x}$, which implies $v_{\mu}\left(\Phi_{x}\right) \leq x$. This finishes the proof.

In this part, we give enough settings on $\left(\ell_{M}(\lambda)\right)_{\mu}$ so that the Kannan $\mu$ - nonexpansive mapping defined on it contains a fixed point.

Theorem 21. Let $\left(\ell_{M}(\lambda)\right)_{\mu}$ hold the $\mu$-quasinormal property and the $(R)$ property. Assume $\Phi$ is a nonempty, $\mu$-convex, $\mu$-closed, and $\mu$-bounded subset of $\left(\ell_{M}(\lambda)\right)_{\mu}$. If $H: \Phi \longrightarrow \Phi$ is a Kannan $\mu$-nonexpansive mapping, then $H$ has a fixed point.

Proof. Let $x_{t}=x_{0}+1 / t$, for all $t \geq 1$, where $x_{0}=\inf \{\mu(\beta-H(\beta)): \beta \in \Phi\}$. We have for each $t \geq 1$ that $W_{x_{t}}=\left\{\beta \in \Phi: \mu(\beta-H(\beta)) \leq x_{t}\right\} \neq \varnothing$. Suppose $\Phi_{x_{t}}$ explained as in Lemma 3. Clearly, $\left\{\Phi_{x_{t}}\right\}$ is a decreasing sequence of nonempty $\mu$-bounded, $\mu$-closed, and $\mu$-convex subsets of $\Phi$. The property $(R)$ gives that $\Phi_{\infty}=\cap_{t \geq 1} \Phi_{x_{t}} \neq \varnothing$. Let $\beta \in \Phi_{\infty}$, we have $\mu(\beta-H(\beta)) \leq x_{t}$, for every $t \geq 1$. If $t \longrightarrow \infty$, one has $\mu(\beta-H(\beta)) \leq x_{0}$, which implies $\mu(\beta-H(\beta))=x_{0}$. Hence, $W_{x_{0}} \neq \varnothing$. So $x_{0}=0$. Otherwise, $x_{0}>0$ which investigates that $H$ has no fixed point. Assume $\Phi_{x_{0}}$ as defined in Lemma 3. Since $H$ has no fixed point and $\Phi_{x_{0}}$ is $H$-invariant, hence $\Phi_{x_{0}}$ holds more than one point, which gives, $v_{\mu}\left(\Phi_{x_{0}}\right)>0$. By the $\mu$-quasinormal property, one has $\beta \in \Phi_{x_{0}}$ with

$$
\mu(\beta-\eta)<v_{\mu}\left(\Phi_{x_{0}}\right) \leq x_{0},
$$

for every $\eta \in \Phi_{x_{0}}$. By Lemma 3, we have $\Phi_{x_{0}} \subset W_{x_{0}}$. By definition of $\Phi_{x_{0}}$, then $H(\beta) \in W_{x_{0}} \subset \Phi_{x_{0}}$. Obviously, one has

$$
\mu(\beta-H(\beta))<v_{\mu}\left(\Phi_{x_{0}}\right) \leq x_{0},
$$

which contradicts the definition of $x_{0}$. So $x_{0}=0$ this implies that any point in $W_{x_{0}}$ is a fixed point of $H$, i.e., $H$ has a fixed point in $\Phi$.

According to Theorem 19, Theorem 20, and Theorem 21, we obtain the next corollary: 
Corollary 2. If $M$ is a uniformly convex Orlicz function satisfying $\Delta_{2}$-condition. Assume $\Phi$ is a nonempty, $\mu$-convex, $\mu$-closed, and $\mu$-bounded subset of $\left(\ell_{M}(\lambda)\right)_{\mu}$. Suppose $H: \Phi \longrightarrow \Phi$ is a Kannan $\mu$-nonexpansive operator. Then $H$ holds a fixed point.

Example 7. Let $H: \Phi \longrightarrow \Phi$ with $H(\beta)$ $= \begin{cases}\beta / 4, & \mu(\beta) \in[0,1), \\ \beta / 5, & \mu(\beta) \in[1, \infty),\end{cases}$

where $\Phi=\left\{\beta \in\left(\ell_{M}(\lambda)\right)_{\mu}: \beta_{0}=\beta_{1}=0\right\}, \quad$ where $\varrho(\beta)=\sum_{x=0}^{\infty} \lambda_{x}\left|\beta_{x}\right|^{2}$, for every $\beta \in\left(\ell_{M}(\lambda)\right)_{\mu}$. As Example 5, the operator $H$ is Kannan $\mu$-contraction mapping. So it is Kannan $\mu$-nonexpansive operator. Clearly, $\Phi$ is a nonempty, $\mu$-convex, $\mu$-closed and $\mu$-bounded subset of $\left(\ell_{M}(\lambda)\right)_{\mu}$. By Corollary 2 , the operator $H$ has a fixed point in $\Phi$.

\section{Kannan $\Upsilon$ - Contraction Mapping on $S_{\left(\ell_{M}(\lambda)\right)_{\mu}}$}

For any two Banach spaces $\mathfrak{X}$ and $\mathfrak{Y}$, we examine in this section the existence of a fixed point of Kannan $\Upsilon$ - contraction mapping on $S_{\left(\ell_{M}(\lambda)\right)_{u}}$, where $\Upsilon(Q)=M^{-1}$ $\left(\sum_{y=0}^{\infty} \lambda_{y} M\left(\left|s_{y}(Q)\right|\right)\right)$, for all $\mathcal{Q} \in S_{\left(\ell_{M}(\lambda)\right)_{\mu}}(\mathfrak{X}, \mathfrak{Y})$.
Theorem 22. If $M$ is a convex Orlicz function satisfying $\Delta_{2}$-condition and $\ln \left(M\left(e^{x}\right)\right)$ is convex, then $\left(S_{\left(\ell_{M}(\lambda)\right)_{\mu}}, Y\right)$ is a prequasi Banach operator ideal, ${ }^{M}$ where $\Upsilon(Q)=\mu\left(\left(s_{y}(Q)\right)_{y=0}^{\infty}\right)$.

Proof. As Theorem 5, the space $\left(\ell_{M}(\lambda)\right)_{\mu}$ is a premodular (sss). Therefore, from Theorem 3, one has $\Upsilon(Q)=\mu\left(\left(s_{y}(Q)\right)_{y=0}^{\infty}\right)$ is a prequasi norm on $S_{\left(\ell_{M}(\lambda)\right)_{\mu}}$. Suppose $Q_{r} \in S_{\left(\ell_{M}(\lambda)\right)_{\mu}}(\mathfrak{X}, \mathfrak{Y})$ is a Cauchy sequence. As $\mathscr{B}(\mathfrak{X}, \mathfrak{Y}) \supseteq S_{\left(\ell_{M}(\lambda)\right)_{\mu}}(\mathfrak{X}, \mathfrak{Y})$, one obtains

$$
\begin{aligned}
\Upsilon\left(Q_{r}-Q_{t}\right) & =\mu\left(\left(s_{y}\left(Q_{r}-Q_{t}\right)\right)_{y=0}^{\infty}\right) \\
& \geq \mu\left(s_{0}\left(Q_{r}-Q_{t}\right), 0,0,0, \ldots,\right)=M^{-1}\left(\lambda_{0}\left\|Q_{r}-Q_{t}\right\|\right) .
\end{aligned}
$$

Hence $\left(Q_{r}\right)_{r \in \mathscr{X}^{+}}$is a Cauchy sequence in $\mathscr{B}(\mathfrak{X}, \mathfrak{Y})$. Since $\mathscr{B}(\mathfrak{X}, \mathfrak{Y})$ is a Banach space, so there is $Q \in \mathscr{B}(\mathfrak{X}, \mathfrak{Y})$ with $\lim _{r \longrightarrow \infty}\left\|Q_{r}-Q\right\|=0$. Since $\left(s_{y}\left(Q_{r}\right)\right)_{y=0}^{\infty} \in\left(\ell_{M}(\lambda)\right)_{\mu}$, for every $r \in \mathscr{Z}^{+}$. We have

$$
\begin{aligned}
\Upsilon(Q) & =\mu\left(\left(s_{y}(Q)\right)_{y=0}^{\infty}\right)=\mu\left(\left(s_{y}\left(Q-Q_{r}+Q_{r}\right)\right)_{y=0}^{\infty}\right) \leq \mu\left(\left(s_{[y / 2]}\left(Q-Q_{r}\right)\right)_{y=0}^{\infty}\right)+\mu\left(\left(s_{[y / 2]}\left(Q_{r}\right)_{y=0}^{\infty}\right)\right) \\
& \leq \mu\left(\left(\left\|Q_{r}-Q\right\|\right)_{y=0}^{\infty}\right)+2 \mu\left(\left(s_{y}\left(Q_{r}\right)_{y=0}^{\infty}\right)\right)<\epsilon .
\end{aligned}
$$

Therefore, $\quad\left(s_{y}(Q)\right)_{y=0}^{\infty} \in\left(\ell_{M}(\lambda)\right)_{\mu}$, this implies $Q \in S_{\left(\ell_{M}(\lambda)\right)_{\mu}}(\mathfrak{X}, \mathfrak{Y})$.

Theorem 23. If $M$ is a convex Orlicz function satisfying $\Delta_{2}$-condition and $\ln \left(M\left(e^{x}\right)\right)$ is convex, then $\left(S_{\left(\ell_{M}(\lambda)\right)_{\mu}}, \Upsilon\right)$ is a prequasi closed operator ideal, where $\Upsilon(Q)=\mu\left(\left(s_{y}(Q)\right)_{y=0}^{\infty}\right)$.

Proof. As Theorem 5, the space $\left(\ell_{M}(\lambda)\right)_{\mu}$ is a premodular (sss). Therefore, from Theorem 3, one has $\Upsilon(Q)=\mu\left(\left(s_{y}(Q)\right)_{y=0}^{\infty}\right)$ is a prequasi norm on $S_{\left(\ell_{M}(\lambda)\right)_{\mu}}$. Assume $Q_{r} \in S_{\left(\ell_{M}(\lambda)\right)_{\mu}}(\mathfrak{X}, \mathfrak{Y})$, for every $r \in \mathscr{Z}^{+}$and $\lim _{r \rightarrow \infty} \Upsilon\left(Q_{r}-Q\right)=0$. Hence, there is $\varsigma>0$ and since $\mathscr{B}(\mathfrak{X}, \mathfrak{Y}) \supseteq S_{\left(\ell_{M}(\lambda)\right)_{\mu}}(\mathfrak{X}, \mathfrak{Y})$, we get

$$
\begin{aligned}
\Upsilon\left(Q_{r}-Q\right) & =\mu\left(\left(s_{y}\left(Q_{r}-Q\right)\right)_{y=0}^{\infty}\right) \geq \mu\left(s_{0}\left(Q_{r}-Q\right), 0,0,0, \ldots\right) \\
& =M^{-1}\left(\lambda_{0}\left\|Q_{r}-Q\right\|\right) .
\end{aligned}
$$

Hence $\left(Q_{r}\right)_{r \in \mathscr{X}^{+}}$is convergent in $\mathscr{B}(\mathfrak{X}, \mathfrak{Y})$. i.e., $\lim _{r \rightarrow \infty}\left\|Q_{r}-Q\right\|=0$ and while $\left(s_{y}\left(Q_{r}\right)\right)_{y=0}^{\infty} \in\left(\ell_{M}(\lambda)\right)_{\mu}$, for all $r \in \mathscr{Z}^{+}$and $\left(\ell_{M}(\lambda)\right)_{\mu}$ is a premodular (sss). Hence, we have

$$
\begin{aligned}
\Upsilon(Q) & =\mu\left(\left(s_{y}(Q)\right)_{y=0}^{\infty}\right)=\mu\left(\left(s_{y}\left(Q-Q_{r}+Q_{r}\right)\right)_{y=0}^{\infty}\right) \\
& \leq \mu\left(\left(s_{[y / 2]}\left(Q-Q_{r}\right)\right)_{y=0}^{\infty}\right)+\mu\left(\left(s_{[y / 2]}\left(Q_{r}\right)_{y=0}^{\infty}\right)\right) \\
& \leq \mu\left(\left(\left\|Q_{r}-Q\right\|\right)_{y=0}^{\infty}\right)+2 \mu\left(\left(s_{y}\left(Q_{r}\right)_{y=0}^{\infty}\right)\right)<\epsilon,
\end{aligned}
$$

we have $\left(s_{y}(Q)\right)_{y=0}^{\infty} \in\left(\ell_{M}(\lambda)\right)_{\mu}$, then $Q \in S_{\left(\ell_{M}(\lambda)\right)}$ ${ }_{\mu}(\mathfrak{X}, \mathfrak{Y})$.

Definition 15. A prequasi norm $\Upsilon$ on the ideal $S_{\left(\ell_{M}(\lambda)\right) \text {, }}$ where $\Upsilon(Q)=\mu\left(\left(s_{y}(Q)\right)_{y=0}^{\infty}\right)$, satisfies the Fatou property if for any sequence $\left\{Q_{y}\right\}_{y \in \mathscr{I}^{+}} \subseteq S_{\left(\ell_{M}(\lambda)\right)_{Y}}(\mathfrak{X}, \mathfrak{Y})$ with $\lim _{y \rightarrow \infty} \Upsilon\left(Q_{y}-Q\right)=0$ and any $V \in S_{\left(\ell_{M}(\lambda)\right)_{\mu}}(\mathfrak{X}, \mathfrak{Y})$, then $\Upsilon(V-Q) \leq \sup _{y} \inf _{j \geq y} \mu\left(V-Q_{j}\right)$.

Theorem 25. The prequasinorm $\Upsilon(Q)=M^{-1}$ $\left(\sum_{y=0}^{\infty} \lambda_{y} M\left(s_{y}(Q)\right)\right)$, for all $Q \in S_{\left(\ell_{M}(\lambda)\right)_{\mu}}(\mathfrak{X}, \mathfrak{Y})$ does not satisfy the Fatou property, if $M$ is a convex Orlicz function satisfying $\Delta_{2}$-condition and $\ln \left(M\left(e^{x}\right)\right)$ is convex.

Proof. Assume the settings are satisfied and $\left\{Q_{t}\right\}_{t \in \mathscr{Z}^{+}} \subseteq S_{\left(\ell_{M}(\lambda)\right)_{u}}(\mathfrak{X}, \mathfrak{Y}) \quad$ with $\quad \lim _{t \rightarrow \infty} \Upsilon\left(Q_{t}-Q\right)=0$. Since the space $S_{\left(\ell_{M}(\lambda)\right)_{\mu}}$ is a prequasi closed ideal, then, 
$Q \in S_{\left(\ell_{M}(\lambda)\right)_{\mu}}(\mathfrak{X}, \mathfrak{Y})$. Hence, for any $V \in S_{\left(\ell_{M}(\lambda)\right)_{\mu}}(\mathfrak{X}, \mathfrak{Y})$, we have

$$
\begin{aligned}
\Upsilon(V-Q) & =M^{-1}\left(\sum_{y=0}^{\infty} \lambda_{y} M\left(s_{y}(V-Q)\right)\right) \leq M^{-1}\left(\sum_{y=0}^{\infty} \lambda_{y} M\left(s_{[y / 2]}\left(V-Q_{j}\right)\right)\right)+M^{-1}\left(\sum_{y=0}^{\infty} \lambda_{y} M\left(s_{[[y / 2]]}\left(Q_{j}-Q\right)\right)\right) \\
& \leq 2 \sup _{t} \inf _{j \geq t} M^{-1}\left(\sum_{y=0}^{\infty} \lambda_{y} M\left(s_{y}\left(V-Q_{j}\right)\right)\right) .
\end{aligned}
$$

Hence, $\Upsilon$ does not satisfy the Fatou property.

Definition 16. An operator P: $S_{\left(\ell_{M}(\lambda)\right)_{\mu}}(\mathfrak{X}, \mathfrak{Y})$ $\longrightarrow S_{\left(\ell_{M}(\lambda)\right)_{\mu}}(\mathfrak{X}, \mathfrak{Y})$ is called a Kannan $\Upsilon$-Lipschitzian, if there exists $\nu \geq 0$, so that for every $Q, T \in S_{\left(\ell_{M}(\lambda)\right)_{\mu}}(\mathfrak{X}, \mathfrak{Y})$, we have

$\Upsilon(P Q-P T) \leq \nu(\Upsilon(P Q-Q)+\Upsilon(P T-T))$.

(1) If $v \in[0,1 / 2)$, the operator $P$ is said to be Kannan $\Upsilon$-contraction.

(2) If $v=1 / 2$, the operator $P$ is said to be Kannan $\Upsilon$-nonexpansive.

Definition 17. An operator $P: S_{\left(\ell_{M}(\lambda)\right)_{\mu}}(\mathfrak{X}, \mathfrak{Y}) \longrightarrow$ $S_{\left(\ell_{M}(\lambda)\right)_{\mu}}(\mathfrak{X}, \mathfrak{Y})$ is said to be $\Upsilon$-sequentially continuous at $V$, if and only if, when $\lim _{t \rightarrow \infty} \Upsilon\left(Q_{t}-V\right)=0$, then $\lim _{t \rightarrow \infty} \Upsilon\left(P Q_{t}-P V\right)=0$.

Theorem 26. If $M$ is a convex Orlicz function satisfying $\Delta_{2}$-condition and $\ln \left(M\left(e^{x}\right)\right)$ is conve, and
$P: S_{\left(\ell_{M}(\lambda)\right)_{\mu}}(\mathfrak{X}, \mathfrak{Y}) \longrightarrow S_{\left(\ell_{M}(\lambda)\right)_{\mu}}(\mathfrak{X}, \mathfrak{Y}) . \quad$ The point $T \in S_{\left(\ell_{M}(\lambda)\right)_{\gamma}}(\mathfrak{X}, \mathfrak{Y})$ is the unique fixed point of $P$, when the following conditions are satisfied:

(i) $P$ is Kannan $Y$-contraction mapping,

(ii) $P$ is $\Upsilon$-sequentially continuous at a point $T \in S_{\left(\ell_{M}(\lambda)\right)_{\mu}}(\mathfrak{X}, \mathfrak{Y})$,

(iii) There exists $V \in S_{\left(\ell_{M}(\lambda)\right)_{U}}(\mathfrak{X}, \mathfrak{Y})$ so that the sequence of iterates $\left\{P^{t} V\right\}$ has a subsequence $\left\{P^{t_{j}} V\right\}$ converging to $T$.

Proof. Let the conditions be verified. If $T$ is not a fixed point of $P$, then $P T \neq T$. From the conditions (ii) and (iii), we have

$$
\begin{array}{r}
\lim _{t_{j} \longrightarrow \infty} \Upsilon\left(P^{t_{j}} V-T\right)=0, \\
\lim _{t_{j} \longrightarrow \infty} \Upsilon\left(P^{t_{j}+1} V-P T\right)=0 .
\end{array}
$$

Since $P$ is Kannan $\Upsilon$-contraction mapping, one can see

$$
\begin{aligned}
0<\Upsilon(P T-T) & =\Upsilon\left(\left(P T-P^{t_{j}+1} V\right)+\left(P^{t_{j}} V-T\right)+\left(P^{t_{j}+1} V-P^{t_{j}} V\right)\right) \\
& \leq 2 \Upsilon\left(P^{t_{j}+1} V-P T\right)+4 \Upsilon\left(P^{t_{j}} V-T\right)+4 \nu\left(\frac{v}{1-v}\right)^{t_{j}-1} \Upsilon(P V-V) .
\end{aligned}
$$

Since $t_{j} \longrightarrow \infty$, we have a contradiction. Hence, $T$ is a fixed point of $P$. To prove the uniqueness of the fixed point $T$. Let we have two different fixed points $T, U \in S_{\left(\ell_{M}(\lambda)\right)_{\mu}}(\mathfrak{X}, \mathfrak{Y})$ of $P$. Therefore, one has

$$
\begin{aligned}
\Upsilon(T-U) & \leq \Upsilon(P T-P U) \\
& \leq \nu(\Upsilon(P T-T)+\Upsilon(P U-U))=0 .
\end{aligned}
$$

Hence, $T=U$.

Example 8. Assume P: $S_{\left(\ell_{M}(\lambda)\right)_{\mu}}(\mathfrak{X}, \mathfrak{Y}) \longrightarrow S_{\left(\ell_{M}(\lambda)\right)_{Y}}(\mathfrak{X}, \mathfrak{Y})$, where $\Upsilon(Q)=\sum_{y=0}^{\infty} \lambda_{y} \sqrt{s_{y}(Q)}$, for every $Q \in S_{\left(\ell_{M}(\lambda)\right)_{\mu}}(\mathfrak{X}, \mathfrak{Y})$ and

$$
P(Q)= \begin{cases}\frac{Q}{26}, & \Upsilon(Q) \in[0,1), \\ \frac{Q}{37}, & \Upsilon(Q) \in[1, \infty) .\end{cases}
$$

As for every $Q_{1}, Q_{2} \in S_{\left(\ell_{M}(\lambda)\right)_{\mu}}$ with $\Upsilon\left(Q_{1}\right), \Upsilon\left(Q_{2}\right)$ $\in[0,1)$, one has

$$
\begin{aligned}
\Upsilon\left(P Q_{1}-P Q_{2}\right) & =\Upsilon\left(\frac{Q_{1}}{26}-\frac{Q_{2}}{26}\right) \leq \frac{2}{5}\left(\Upsilon\left(\frac{25 Q_{1}}{26}\right)+\Upsilon\left(\frac{25 Q_{2}}{26}\right)\right) \\
& =\frac{2}{5}\left(\Upsilon\left(P Q_{1}-Q_{1}\right)+\Upsilon\left(P Q_{2}-Q_{2}\right)\right) .
\end{aligned}
$$



we get

For each $Q_{1}, Q_{2} \in S_{\left(\ell_{M}(\lambda)\right)_{\mu}}$ with $\Upsilon\left(Q_{1}\right), \Upsilon\left(Q_{2}\right) \in[1, \infty)$,

$$
\Upsilon\left(P Q_{1}-P Q_{2}\right)=\Upsilon\left(\frac{Q_{1}}{37}-\frac{Q_{2}}{37}\right) \leq \frac{1}{3}\left(\Upsilon\left(\frac{36 Q_{1}}{37}\right)+\Upsilon\left(\frac{36 Q_{2}}{37}\right)\right)=\frac{1}{3}\left(\Upsilon\left(P Q_{1}-Q_{1}\right)+\Upsilon\left(P Q_{2}-Q_{2}\right)\right)
$$

For each $Q_{1}, Q_{2} \in S_{\left(\ell_{M}(\lambda)\right)_{\mu}}$ with $\Upsilon\left(Q_{1}\right) \in[0,1)$ and $\Upsilon\left(Q_{2}\right) \in[1, \infty)$, one can see

$$
\begin{aligned}
\Upsilon\left(P Q_{1}-P Q_{2}\right) & =\Upsilon\left(\frac{Q_{1}}{26}-\frac{Q_{2}}{37}\right) \leq \frac{2}{5} \Upsilon\left(\frac{25 Q_{1}}{26}\right)+\frac{1}{3} \Upsilon\left(\frac{36 Q_{2}}{37}\right) \leq \frac{2}{5}\left(\Upsilon\left(\frac{25 Q_{1}}{26}\right)+\Upsilon\left(\frac{36 Q_{2}}{37}\right)\right) \\
& =\frac{2}{5}\left(\Upsilon\left(P Q_{1}-Q_{1}\right)+\Upsilon\left(P Q_{2}-Q_{2}\right)\right) .
\end{aligned}
$$

So, the operator $Q$ is Kannan $\Upsilon$-contraction and $P^{t}(Q)=$ $\left\{Q / 26^{t}, \quad \Upsilon(Q) \in[0,1)\right.$

$\left\{\mathrm{Q} / 37^{t}, \quad \Upsilon(Q) \in[1, \infty)\right.$.

Clearly, $P$ is $\Upsilon$-sequentially continuous at the zero operator $\Theta \in S_{\left(\ell_{M}(\lambda)\right)_{\mu}}$ and $\left\{P^{t} Q\right\}$ has a subsequence $\left\{P^{t_{j}} Q\right\}$ converging to $\Theta$. From Theorem 27, the zero operator $\Theta \in S_{\left(\ell_{M}(\lambda)\right)_{\mu}}$ is the unique fixed point of $P$. Suppose with $\lim _{t \rightarrow \infty} \Upsilon\left(Q^{(t)}-Q^{(0)}\right)=0-b \pm \sqrt{b^{2}-4 a c} / 2 a$, where $Q^{(0)} \in S_{\left(\ell_{M}(\lambda)\right)_{\mu}}$ with $\Upsilon\left(Q^{(0)}\right)=1$. From the continuously of the prequasi norm $\Upsilon$, one has

$$
\begin{aligned}
\lim _{t \rightarrow \infty} \Upsilon\left(P Q^{(t)}-P Q^{(0)}\right) & =\lim _{t \longrightarrow \infty} \Upsilon\left(\frac{Q^{(t)}}{26}-\frac{Q^{(0)}}{37}\right) \\
& =\Upsilon\left(\frac{11 Q^{(0)}}{962}\right)>0 .
\end{aligned}
$$

So $P$ is not $\Upsilon$-sequentially continuous at $Q^{(0)}$. This implies the operator $P$ is not continuous at $Q^{(0)}$.

Example 9. Suppose P: $S_{\left(\ell_{M}(\lambda)\right)_{\mu}}(\mathfrak{X}, \mathfrak{Y}) \longrightarrow S_{\left(\ell_{M}(\lambda)\right)_{Y}}(\mathfrak{X}, \mathfrak{Y})$, where $\Upsilon(Q)=\sqrt{\sum_{y=0}^{\infty} \lambda_{y}\left(s_{y}(Q)\right)^{2}}, \quad$ for every $Q \in S_{\left(\ell_{M}(\lambda)\right)_{Y}}(\mathfrak{X}, \mathfrak{Y})$ and

$$
P(Q)= \begin{cases}\frac{Q}{5}, & \Upsilon(Q) \in[0,1), \\ \frac{Q}{6}, & \Upsilon(Q) \in[1, \infty) .\end{cases}
$$

As for each $Q_{1}, Q_{2} \in S_{\left(\ell_{M}(\lambda)\right)_{\mu}}$ with $\Upsilon\left(Q_{1}\right), \Upsilon\left(Q_{2}\right) \in$ $[0,1)$, one can see

$$
\begin{aligned}
\Upsilon\left(P Q_{1}-P Q_{2}\right) & =\Upsilon\left(\frac{Q_{1}}{5}-\frac{Q_{2}}{5}\right) \leq \frac{\sqrt{2}}{4}\left(\Upsilon\left(\frac{4 Q_{1}}{5}\right)+\Upsilon\left(\frac{4 Q_{2}}{5}\right)\right) \\
& =\frac{\sqrt{2}}{4}\left(\Upsilon\left(P Q_{1}-Q_{1}\right)+\Upsilon\left(P Q_{2}-Q_{2}\right)\right) .
\end{aligned}
$$

For every $Q_{1}, Q_{2} \in S_{\left(\ell_{M}(\lambda)\right)_{\mu}}$ with $\Upsilon\left(Q_{1}\right), \Upsilon\left(Q_{2}\right) \in[1, \infty)$, this implies

$$
\begin{aligned}
\Upsilon\left(P Q_{1}-P Q_{2}\right) & =\Upsilon\left(\frac{Q_{1}}{6}-\frac{Q_{2}}{6}\right) \leq \frac{\sqrt{2}}{5}\left(\Upsilon\left(\frac{5 Q_{1}}{6}\right)+\Upsilon\left(\frac{5 Q_{2}}{6}\right)\right) \\
& =\frac{\sqrt{2}}{5}\left(\Upsilon\left(P Q_{1}-Q_{1}\right)+\Upsilon\left(P Q_{2}-Q_{2}\right)\right) .
\end{aligned}
$$

For each $Q_{1}, Q_{2} \in S_{\left(\ell_{M}(\lambda)\right)_{\mu}}$ with $\Upsilon\left(Q_{1}\right) \in[0,1)$ and $\Upsilon\left(Q_{2}\right) \in[1, \infty)$, one obtains

$$
\begin{aligned}
\Upsilon\left(P Q_{1}-P Q_{2}\right) & =\Upsilon\left(\frac{Q_{1}}{5}-\frac{Q_{2}}{6}\right) \leq \frac{\sqrt{2}}{4} \Upsilon\left(\frac{4 Q_{1}}{5}\right)+\frac{\sqrt{2}}{5} \Upsilon\left(\frac{5 Q_{2}}{6}\right) \\
& \leq \frac{\sqrt{2}}{4}\left(\Upsilon\left(\frac{4 Q_{1}}{5}\right)+\Upsilon\left(\frac{5 Q_{2}}{6}\right)\right) \\
& =\frac{\sqrt{2}}{4}\left(\Upsilon\left(P Q_{1}-Q_{1}\right)+\Upsilon\left(P Q_{2}-Q_{2}\right)\right) .
\end{aligned}
$$

So, the operator $Q$ is Kannan $\Upsilon$-contraction and. $P^{t}(Q)= \begin{cases}Q / 5^{t}, & \Upsilon(Q) \in[0,1) \\ Q / 6^{t}, & \Upsilon(Q) \in[1, \infty)\end{cases}$

Evidently, $P$ is $\Upsilon$-sequentially continuous at the zero operator $\Theta \in S_{\left(\ell_{M}(\lambda)\right)_{\mu}}$ and $\left\{P^{t} Q\right\}$ has a subsequence $\left\{P^{t_{j}} Q\right\}$ converging to $\Theta$. From Theorem 27, the zero operator $\Theta \in S_{\left(\ell_{M}(\lambda)\right)_{\mu}}$ is the unique fixed point of $P$. Suppose 
$\left\{Q^{(t)}\right\} \subseteq S_{\left(\ell_{M}(\lambda)\right)_{\mu}}$ with $\lim _{t \rightarrow \infty} \Upsilon\left(Q^{(t)}-Q^{(0)}\right)=0$, where $Q^{(0)} \in S_{\left(\ell_{M}(\lambda)\right)_{\mu}}$ with $\Upsilon\left(Q^{(0)}\right)=1$. From the continuously of the prequasi norm $Y$, one has

$$
\begin{aligned}
\lim _{t \longrightarrow \infty} \Upsilon\left(P Q^{(t)}-P Q^{(0)}\right) & =\lim _{t \longrightarrow \infty} \Upsilon\left(\frac{Q^{(t)}}{5}-\frac{Q^{(0)}}{6}\right) \\
& =\Upsilon\left(\frac{Q^{(0)}}{30}\right)>0 .
\end{aligned}
$$

So $P$ is not $\Upsilon$-sequentially continuous at $Q^{(0)}$. Hence, the operator $P$ is not continuous at $Q^{(0)}$.

\section{Applications on Summable Equations}

We investigate here a solution to (101), which studied by many authors (see [27-29]), in $\left(\ell_{M}(\lambda)\right)_{\mu}$.

$$
\beta_{x}=r_{x}+\sum_{y=0}^{\infty} D(x, y) h\left(y, \beta_{y}\right) .
$$

Suppose $H:\left(\ell_{M}(\lambda)\right)_{\mu} \longrightarrow\left(\ell_{M}(\lambda)\right)_{\mu}$ constructed by

$$
H\left(\beta_{x}\right)_{x \in \mathscr{L}^{+}}=\left(r_{x}+\sum_{y=0}^{\infty} D(x, y) h\left(y, \beta_{y}\right)\right)_{x \in \mathscr{L}^{+}} .
$$

Theorem 27. If $M$ is a convex Orlicz function satisfying $\Delta_{2}$-condition and $\ln \left(M\left(e^{x}\right)\right)$ is convex, $D: \mathscr{Z}^{+2} \longrightarrow \mathbf{R}$, $h: \mathscr{Z}^{+} \times \mathbf{R} \longrightarrow \mathbf{R}, r: \mathscr{Z}^{+} \longrightarrow \mathbf{R}$, and for all $x \in \mathscr{Z}^{+}$, there exists $v \in[0,1 / 2)$, with

$$
\begin{aligned}
& M\left(\left|\sum_{y \in \mathscr{E}^{+}} D(x, y)\left(h\left(y, \beta_{y}\right)-h\left(y, \eta_{y}\right)\right)\right|\right) \\
& \leq M(v)\left[M\left(\left|r_{x}-\beta_{x}+\sum_{y=0}^{\infty} D(x, y) f\left(y, \beta_{y}\right)\right|\right)+M\left(\left|r_{x}-\eta_{x}+\sum_{y=0}^{\infty} D(x, y) f\left(y, \eta_{y}\right)\right|\right)\right],
\end{aligned}
$$

then equation (101) hold a solution in $\left(\ell_{M}(\lambda)\right)_{\mu}$, where Proof. Suppose the setups are verified. We have $\mu(\beta)=M^{-1}\left(\sum_{x=0}^{\infty} M\left(\left|\beta_{x}\right|\right)\right)$, for every $\beta \in \ell_{M}(\lambda)$.

$$
\begin{aligned}
\mu(H \beta-H \eta)=M^{-1}\left(\sum_{x \in \mathscr{I}^{+}} \lambda_{x} M\left(\left|H \beta_{x}-H \eta_{x}\right|\right)\right)= & M^{-1}\left(\sum_{x \in \mathscr{T}^{+}} \lambda_{x} M\left(\left|\sum_{m \in \mathscr{E}^{+}} D(x, y)\left[h\left(y, \beta_{y}\right)-h\left(y, \eta_{y}\right)\right]\right|\right)\right) \\
\leq & v M^{-1}\left(\sum_{x \in \mathscr{X}^{+}} \lambda_{x} M\left(\left|r_{x}-\beta_{x}+\sum_{m=0}^{\infty} D(x, y) h\left(y, \beta_{y}\right)\right|\right)\right) \\
& +v M^{-1}\left(\sum_{x \in \mathscr{F}^{+}} \lambda_{x} M\left(\left|r_{x}-\eta_{x}+\sum_{m=0}^{\infty} D(x, y) h\left(y, \eta_{y}\right)\right|\right)\right) \\
= & \nu(\mu(H \beta-\beta)+\mu(H \eta-\eta)) .
\end{aligned}
$$

In view of Theorem 11, there exists a unique solution of equation (101) in $\left(\ell_{M}(\lambda)\right)_{\mu}$.

Example 10. For the space $\left(\ell_{M}(\lambda)\right)_{\mu}$, where $\mu(\beta)=\sqrt[4]{\sum_{x \in \mathscr{Z}^{+}}\left|\beta_{x}\right|^{4}}$, for all $\beta \in \ell_{M}(\lambda)$. Assume the summable equations are defined as

$$
\beta_{x}=e^{-(3 x+6)}+\sum_{y=0}^{\infty}(-1)^{x}\left(\frac{\beta_{x}}{x^{2}+y^{2}+1}\right)^{v} \sin y,
$$

where $v>2$ and let $H:\left(\ell_{M}(\lambda)\right)_{\mu} \longrightarrow\left(\ell_{M}(\lambda)\right)_{\mu}$ is defined by

$$
H\left(\beta_{x}\right)_{x \in \mathscr{I}^{+}}=\left(e^{-(3 x+6)}+\sum_{y=0}^{\infty}(-1)^{x}\left(\frac{\beta_{x}}{x^{2}+y^{2}+1}\right)^{v} \sin y\right)_{x \in \mathscr{I}^{+}} .
$$


We have

$$
\begin{aligned}
& \left|\sum_{y=0}^{\infty}(-1)^{x}\left(\frac{\beta_{x}}{x^{2}+y^{2}+1}\right)^{v}(\sin y-\sin y)\right|^{4} \\
& \leq \frac{1}{81}\left[\left|e^{-(3 x+6)}-\beta_{x}+\sum_{y=0}^{\infty}(-1)^{x}\left(\frac{\beta_{x}}{x^{2}+y^{2}+1}\right)^{v} \sin y\right|^{4}+\left|e^{-(3 x+6)}-\eta_{x}+\sum_{y=0}^{\infty}(-1)^{x}\left(\frac{\eta_{x}}{x^{2}+y^{2}+1}\right)^{v} \sin y\right|^{4}\right] .
\end{aligned}
$$

By Theorem 27, the summable equations (105) have one solution in $\left(\ell_{M}(\lambda)\right)_{\mu}$.
Theorem 30. If $M$ is a concave Orlicz function, $D: \mathscr{Z}^{+2} \longrightarrow \mathbf{R}, h: \mathscr{Z}^{+} \times \mathbf{R} \longrightarrow \mathbf{R}, r: \mathscr{Z}^{+} \longrightarrow \mathbf{R}$, and for every $x \in \mathscr{Z}^{+}$, there exists $v \in[0,1 / 2)$, with

$$
\begin{aligned}
& M\left(\left|\sum_{y \in \mathscr{L}^{+}} D(x, y)\left(h\left(y, \beta_{y}\right)-h\left(y, \eta_{y}\right)\right)\right|\right) \\
& \leq \nu\left[M\left(\left|r_{x}-\beta_{x}+\sum_{y=0}^{\infty} D(x, y) f\left(y, \beta_{y}\right)\right|\right)+M\left(\left|r_{x}-\eta_{x}+\sum_{y=0}^{\infty} D(x, y) f\left(y, \eta_{y}\right)\right|\right)\right]
\end{aligned}
$$

then equation (101) contains one solution in $\left(\ell_{M}(\lambda)\right)_{\mu}$, where Proof. Suppose the setups are verified. One has $\mu(\beta)=\sum_{x=0}^{\infty} M\left(\left|\beta_{x}\right|\right)$, for each $\beta \in \ell_{M}(\lambda)$.

$$
\begin{aligned}
\mu(H \beta-H \eta) & \left.=\sum_{x \in \mathscr{L}^{+}} \lambda_{x} M\left(\left|H \beta_{x}-H \eta_{x}\right|\right)\right)=\sum_{x \in \mathscr{Z}^{+}} \lambda_{x} M\left(\left|\sum_{y \in \mathscr{Z}^{+}} D(x, y)\left(h\left(y, \beta_{y}\right)-h\left(y, \eta_{y}\right)\right)\right|\right) \\
& \leq \nu\left[\sum_{x \in \mathscr{Z}^{+}} \lambda_{x} M\left(\left|r_{x}-\beta_{x}+\sum_{y=0}^{\infty} D(x, y) h\left(y, \beta_{y}\right)\right|\right)+\sum_{x \in \mathscr{Z}^{+}} \lambda_{x} M\left(\left|r_{x}-\eta_{x}+\sum_{y=0}^{\infty} D(x, y) h\left(y, \eta_{y}\right)\right|\right)\right] \\
& =\nu(\mu(H \beta-\beta)+\mu(H \eta-\eta)) .
\end{aligned}
$$

In view of Theorem 12 , there exists a unique solution of equation (101) in $\left(\ell_{M}(\lambda)\right)_{\mu}$.

Example 11. For the space $\left(\ell_{M}(\lambda)\right)_{\mu}$, where $\mu(\beta)=\sum_{x \in \mathscr{Z}^{+}} \sqrt[3]{\left|\beta_{x}\right|}$, for every $\beta \in \ell_{M}(\lambda)$. Assume the summable equations

$$
\beta_{x}=e^{-(3 x+6)}+\sum_{y=0}^{\infty}(-1)^{x+y}\left(\frac{e^{\left|\beta_{x}\right|}}{x^{2}+y^{2}+1}\right)^{v},
$$

where $v>2$ and let $H:\left(\ell_{M}(\lambda)\right)_{\mu} \longrightarrow\left(\ell_{M}(\lambda)\right)_{\mu}$ is defined by

$$
H\left(\beta_{x}\right)_{x \in \mathscr{E}^{+}}=\left(e^{-(3 x+6)}+\sum_{y=0}^{\infty}(-1)^{x+y}\left(\frac{e^{\left|\beta_{x}\right|}}{x^{2}+y^{2}+1}\right)^{v}\right)_{x \in \mathscr{Z}^{+}} \text {. }
$$

It is easy to see that

$$
\begin{aligned}
& \left|\sum_{y=0}^{\infty}(-1)^{x}\left(\frac{e^{\left|\beta_{x}\right|}}{x^{2}+y^{2}+1}\right)^{v}\left((-1)^{y}-(-1)^{y}\right)\right|^{1 / 3} \\
& \leq \frac{1}{3}\left[\left|e^{-(3 x+6)}-\beta_{x}+\sum_{y=0}^{\infty}(-1)^{x+y}\left(\frac{e^{\left|\beta_{x}\right|}}{x^{2}+y^{2}+1}\right)^{v}\right|^{1 / 3}+\left|e^{-(3 x+6)}-\eta_{x}+\sum_{y=0}^{\infty}(-1)^{x+y}\left(\frac{e^{\left|\beta_{x}\right|}}{x^{2}+y^{2}+1}\right)^{v}\right|^{1 / 3}\right] .
\end{aligned}
$$


By Theorem 30, the summable equation (105) has an unique solution in $\left(\ell_{M}(\lambda)\right)_{\mu}$.

Example 12. Given the sequence space $\left(\ell_{M}(\lambda)\right)_{\mu}$, where $\mu(\beta)=\sqrt{\sum_{x \in \mathscr{Z}^{+}}\left|\beta_{x}\right|^{2}}$, for all $\beta \in \ell_{M}(\lambda)$. Consider the summable equations (110), with $x \geq 2$ and $v>2$ and let $H: \Phi \longrightarrow \Phi$, where $\Phi=\left\{\beta \in\left(\ell_{M}(\lambda)\right)_{\mu}: \beta_{0}=\beta_{1}=0\right\}$, defined by

$$
H\left(\beta_{x}\right)_{x \geq 2}=\left(e^{-(3 x+6)}+\sum_{y=0}^{\infty}(-1)^{x+y}\left(\frac{e^{\left|\beta_{x}\right|}}{x^{2}+y^{2}+1}\right)^{v}\right)_{x \geq 2}
$$

Obviously, $\Phi$ is a nonempty, $\mu$-convex, $\mu$-closed, and $\mu$-bounded subset of $\left(\ell_{M}(\lambda)\right)_{\mu}$. It is easy to see that

$$
\begin{aligned}
& \left|\sum_{y=0}^{\infty}(-1)^{x}\left(\frac{e^{\left|\beta_{x}\right|}}{x^{2}+y^{2}+1}\right)^{v}\left((-1)^{y}-(-1)^{y}\right)\right|^{2} \\
& \leq \frac{1}{9}\left[\left|e^{-(3 x+6)}-\beta_{x}+\sum_{y=0}^{\infty}(-1)^{x+y}\left(\frac{e^{\left|\beta_{x}\right|}}{x^{2}+y^{2}+1}\right)^{v}\right|^{2}+\left|e^{-(3 x+6)}-\eta_{x}+\sum_{y=0}^{\infty}(-1)^{x+y}\left(\frac{e^{\left|\eta_{x}\right|}}{x^{2}+y^{2}+1}\right)^{v}\right|^{2}\right] .
\end{aligned}
$$

By Theorem 27 and Corollary 2, the summable equation (110) have a solution in $\Phi$.

\section{Conclusion}

We explored the presence of a fixed point for both Kannan contraction and nonexpansive mappings working on various premodular, which is a generalization of modular, defined by weighted Orlicz sequence space and its prequasi operator ideal. Numerous numerical experiments and practical applications are used to substantiate our findings.

\section{Data Availability}

No data were used to support this study.

\section{Ethical Approval}

This article does not contain any studies with human participants or animals performed by any of the authors.

\section{Conflicts of Interest}

The authors declare that they have no competing interests.

\section{Authors' Contributions}

All the authors contributed equally to the writing of this paper. All the authors read and approved the final manuscript.

\section{Acknowledgments}

This work was funded by the University of Jeddah, Saudi Arabia, under grant No. (UJ-21-DR-92). The authors, therefore, acknowledge with thanks the University technical and financial support.

\section{References}

[1] M. A. Krasnoselskii and Y. B. Rutickii, Convex Functions and Orlicz Spaces, Gorningen, Gorningen, Netherlands, 1961.

[2] W. Orlicz and Ü.. Raume, “(LM)," Bulletin International de l'Academie des Sciences de Cracovie, pp. 93-107, 1936.

[3] J. Lindenstrauss and L. Tzafriri, "On Orlicz sequence spaces," Israel Journal of Mathematics, vol. 10, no. 3, pp. 379-390, 1971.

[4] J. Lindenstrauss and L. Tzafriri, "Classical Banach spaces," I. Sequence spaces," Ergebnisse der Mathematik und ihrer Grenzgebiete, Vol. 92, Springer-Verlag, Berlin, Germany, 1977.

[5] A. Pietsch, Eigenvalues and s-numbers, Cambridge University Press, New York, NY, USA, 1986.

[6] N. Faried and A. A. Bakery, "Small operator ideals formed by s numbers on generalized Cesáro and Orlicz sequence spaces $s$ numbers on generalized Cesàro and Orlicz sequence spaces," Journal of Inequalities and Applications, vol. 2018, no. 1, 357 pages, 2018.

[7] A. A. Bakery and A. R. Abou Elmatty, "Pre-quasi simple Banach operator ideal generated by s-numbers," Journal of Function Spaces, vol. 2020, Article ID 9164781, 11 pages, 2020.

[8] S. Banach, "Sur les opérations dans les ensembles abstraits et leur application aux équations intégrales," Fundamenta Mathematicae, vol. 3, pp. 133-181, 1922.

[9] R. Kannan, "Some results on fixed points-II," The American Mathematical Monthly, vol. 76, no. 4, pp. 405-408, 1969.

[10] S. J. H. Ghoncheh, "Some fixed point theorems for Kannan mapping in the modular spaces," Ciencia e Natura, vol. 37, pp. 462-466, 2015.

[11] H. H. Alsulami, A. Roldan, E. Karapinar, and S. Radenovic, "Some inevitable remarks on Tripled fixed point theorems for mixed monotone Kannan type contractive mappings," Journal of Applied Mathematics, vol. 2014, Article ID 392301, 7 pages, 2014.

[12] E. Karapinar, "Best proximity points of Kannan type cylic weak phi-contractions in ordered metric spaces," Analele Stiintifice ale Universitatii Ovidius Constanta, vol. 20, no. 3, pp. 51-64, 2012.

[13] Ü. Aksoy, E. Karapınar, and İ. Erhan, "Fixed point theorems in complete modular metric spaces and an application to anti- 
periodic boundary value problems," Filomat, vol. 31, no. 17, pp. 5475-5488, 2017.

[14] Ü. Aksoy, E. Karapınar, İ. Erhan, and V. Rakocevic, "Meirkeeler type contractions on modular metric spaces," Filomat, vol. 32, no. 10, pp. 3697-3707, 2018.

[15] A. A. Bakery and O. S. K. Mohamed, "Kannan prequasi contraction maps on nakano sequence spaces," Journal of Function Spaces, vol. 2020, Article ID 8871563, 10 pages, 2020.

[16] S. Reich and A. J. Zaslavski, "Fixed points and convergence results for a class of contractive mappings," Journal of Nonlinear and Variational Analysis, vol. 5, pp. 665-671, 2021.

[17] A. Dehici and N. Redjel, "Some fixed point results for nonexpansive mappings in Banach spaces," Journal of Nonlinear Functional Analysis, vol. 36, 2020.

[18] H. Bendahmane and B. Bendoukha, "Some fixed point results in (p,q)-Metric spaces," Commun Optim Theory, vol. 16, 2020.

[19] H. Nakano, "Modulared sequence spaces," Proceedings of the Japan Academy, vol. 27, pp. 508-512, 1951.

[20] A. A. Bakery and O. K. S. K. Mohamed, "Kannan nonexpansive maps on generalized Cesàro backward difference sequence space of non-absolute type with applications to summable equations," Journal of Inequalities and Applications, vol. 2021, no. 1, p. 103, 2021.

[21] A. Pietsch, Operator Ideals, North-Holland Publishing Company, Amsterdam-New York-Oxford, 1980.

[22] H. P. Mulholland, "On generalizations of Minkowski's inequality in the form of a triangle inequality," Proceedings of the London Mathematical Society, vol. s2-51, pp. 294-307, 1950.

[23] M. Kuczma, An Introduction to the Theory of Functional Equations and Inequalities. Cauchy's Equation and Jensen's Inequality, Uniwersytet Śląski, Polish Scientific Publishers, Warszawa-Kraków-Katowice, 1985.

[24] A. Kamińska, "On uniform convexity of orlicz spaces," Indagationes Mathematicae, vol. 85, no. 1, pp. 27-36, 1982.

[25] G. Albinus, "Normartige Metriken auf metrisierbaren lokalkonvexen topologischen Vektorräumen," Mathematische Nachrichten, vol. 37, no. 3-4, pp. 177-196, 1968.

[26] G. C. Ahuja, T. D. Narang, and S. Trehan, "Best approximation on convex sets in metric linear spaces," Mathematische Nachrichten, vol. 78, no. 1, pp. 125-130, 1977.

[27] P. Salimi, L. Abdul, and N. Hussain, "Modified $\alpha$ - $\psi$-contractive mappings with applications," Fixed Point Theory and Applications, vol. 151, 2013.

[28] R. P. Agarwal, N. Hussain, and M.-A. Taoudi, "Fixed point theorems in ordered Banach spaces and applications to nonlinear integral equations," Abstract and Applied Analysis, vol. 2012, Article ID 245872, 21 pages, 2012.

[29] N. Hussain, A. R. Khan, and R. P. Agarwal, "Krasnosel'skii and Ky Fan type fixed point theorems in ordered Banach spaces," Journal of . Nonlinear Convex Anal, vol. 11, no. 3, pp. 475-489, 2010. 